Noname manuscript No.

(will be inserted by the editor)

\title{
Nonlinear weakly curved rod by $\Gamma$-convergence
}

\author{
Igor Velčić $\dagger \ddagger$
}

the date of receipt and acceptance should be inserted later

\begin{abstract}
We present a nonlinear model of weakly curved rod, namely the type of curved rod where the curvature is of the order of the diameter of the cross-section. We use the approach analogous to the one for rods and curved rods and start from the strain energy functional of three dimensional nonlinear elasticity and do not presuppose any constitutional behavior. To derive the model, by means of $\Gamma$ convergence, we need to propose how is the order of strain energy related to the thickness of the body $h$. We analyze the situation when the strain energy (divided by the order of volume) is of the order $h^{4}$. That is the same approach as the one when Föppl-von Kármán model for plates and the analogous model for rods are obtained. The obtained model is analogous to Marguerre-von Kármán for shallow shells and its linearization is the linear shallow arch model which can be found in the literature.
\end{abstract}

Keywords weakly curved rod · Gamma convergence $\cdot$ shallow arch · asymptotic analysis

Mathematics Subject Classification (2010) $74 \mathrm{~K} 20 \cdot 74 \mathrm{~K} 25$

\section{Introduction}

The study of thin structures is the subject of numerous works in the theory of elasticity. There is a vast literature on the subject of rods, plates and shells (see $[5,8,9])$.

The derivation and justification of the lower dimensional models, equilibrium and dynamic, of rods, curved rods, weakly curved rods, plates and shells in linearized elasticity, by using formal asymptotic expansion, is well established (see [8, 9] and the references therein). In all these approaches one starts from the equations

$\dagger$ BCAM -Basque Center for Applied Mathematics Address Bizkaia Technology Park, 500, 48160 Derio Basque Country - Spain

E-mail: ivelcic@bcamath.org .

$\ddagger$ Faculty of Electrical Engineering and Computer Science, University of Zagreb, Unska 3, 10000 Zagreb, Croatia. 
of three-dimensional linearized elasticity and then via formal asymptotic expansion justify the lower dimensional models. One can also obtain the convergence results. In $[3,4]$ the linear model of weakly curved rod (or as it is called shallow arch) is derived and the convergence result is obtained. We call weakly curved rods or shallow arches those characterized by the fact that the curvature of their centerline has the same order of magnitude as the diameter of the cross section, both being much smaller than their length.

Formal asymptotic expansion is also applied to derive non linear models of rods, plates and shells (see $[8,9,22]$ and the references therein), starting from three-dimensional isotropic elasticity (usually Saint-Venant-Kirchoff material). Hierarchy of the models is obtained, depending on the order of the external loads related to the thickness of the body $h$ (see also [11] for plates).

However, formal asymptotic expansion does not provide us a convergence result. The first convergence result, in deriving lower dimensional models from threedimensional non linear elasticity, is obtained applying $\Gamma$-convergence, very powerful tool introduced by Degiorgi (see $[6,10]$ ). Using $\Gamma$-convergence, elastic string models, membrane plate and membrane shell models are obtained (see $[1,16,17]$ ). It is assumed that the external loads are of order $h^{0}$. The obtained models are different from those ones obtained by the formal asymptotic expansion in the sense that additional relaxation of the energy functional is done.

Recently, hierarchy of models of rods, curved rods, plates and shells is obtained via $\Gamma$ - convergence (see $[12-15,19,24,25,30,31]$ ). Influence of the boundary conditions and the order and the type of the external loads is largely discussed for plates (see $[13,18]$ ). Let us mention that $\Gamma$-convergence results provide us the convergence of the global minimizers of the total energy functional. Recently, compensated compactness arguments are used to obtain the convergence of the stationary points of the energy functional (see $[23,28]$ ).

Here we apply the tools developed for rods, plates and shells to obtain weakly curved rod model by $\Gamma$-convergence. It is assumed that we have free boundary conditions and that the strain energy (divided by the order of volume) is of the order $h^{4}$, where $h$ is the thickness of the rod. This corresponds to the situation when external transversal dead loads are of order $h^{3}$ (see Remark 8). The order $h^{4}$ of the strain energy gives Föppl-von Kármán model for plates, Marguerre-von Kármán model for shallow shells and the analogous model for rods (see $[14,25$, $32]$ ). The obtained model is non linear model of the highest order in the hierarchy of models and its linearization is shallow arch model, obtained in [3, 4] for isotropic, homogenous case (see for comparison Remark $7 \mathrm{~d}$ )). Here we do not presuppose any constitutional behavior and thus work in a more general framework. The main result is stated in Theorem 5 .

Throughout the paper $\bar{A}$ or $\{A\}^{-}$denotes the closure of the set. By a domain we call a bounded open set with Lipschitz boundary. I denotes the identity matrix, by $\mathrm{SO}(3)$ we denote the rotations in $\mathbb{R}^{3}$, by so(3) the set of antisymmetric matrices $3 \times 3$ and $\mathbb{R}_{\mathrm{sym}}^{3 \times 3}$ denotes the set of symmetric matrices. By sym $\mathbf{A}$ we denote the symmetric part of the matrix, $\operatorname{sym} \mathbf{A}=\frac{1}{2}\left(\mathbf{A}+\mathbf{A}^{T}\right) \cdot \boldsymbol{e}_{1}, \boldsymbol{e}_{2}, \boldsymbol{e}_{3}$ are the vectors of the canonical base in $\mathbb{R}^{3}$. By $\nabla_{h}$ we denote $\nabla_{h}=\nabla_{\boldsymbol{e}_{1}}+\frac{1}{h} \nabla_{\boldsymbol{e}_{2}, \boldsymbol{e}_{3}}$. $\|f\|_{C^{1}(\Omega)}$ stands for $C^{1}$ norm of the function $f: \Omega \subset \mathbb{R}^{n} \rightarrow \mathbb{R}$ i.e. $\|f\|_{C^{1}(\Omega)}=$ $\max _{x \in \Omega}|f|+\sum_{i=1}^{n} \max _{x \in \Omega}\left|\partial_{i} f\right|$. $\rightarrow$ denotes the strong convergence and $\rightarrow$ the weak convergence. 


\section{Setting up the problem}

Let $\omega \subset \mathbb{R}^{2}$ be an open set having area equal to $A$ and Lipschitz boundary. For all $h$ such that $0<h \leq 1$ and for given $L$ we define

$$
\omega^{h}=h \omega, \Omega^{h}=(0, L) \times h \omega .
$$

We shall leave out superscript when $h=1$, i.e. $\Omega=\Omega^{1}, \omega=\omega^{1}$. Let us by $\mu(\omega)$ denote

$$
\mu(\omega)=\int_{\omega}\left(x_{2}^{2}+x_{3}^{2}\right) d x_{2} d x_{3} .
$$

Let us choose coordinate axis such that

$$
\int_{\omega} x_{2} d x_{2} d x_{3}=\int_{\omega} x_{3} d x_{2} d x_{3}=\int_{\omega} x_{2} x_{3} d x_{2} d x_{3}=0 .
$$

For every $h$ we define the curve $C^{h}$ of the form

$$
C^{h}=\left\{\theta^{h}\left(x_{1}\right)=\left(x_{1}, \theta_{2}^{h}\left(x_{1}\right), \theta_{3}^{h}\left(x_{1}\right)\right) \in \mathbb{R}^{3}: x_{1} \in(0, L)\right\} .
$$

where $\theta_{k}^{h}\left(x_{1}\right)$, for $k=2,3$, are given functions satisfying $\theta_{k}^{h} \in C^{3}(0, L)$. Let $\left(\boldsymbol{t}^{h}, \boldsymbol{n}^{h}, \boldsymbol{b}^{h}\right)$ be the Frenet trihedron associated with the curve $C^{h}$

$$
\begin{aligned}
\boldsymbol{t}^{h} & =\frac{1}{\sqrt{1+\left(\left(\theta_{2}^{h}\right)^{\prime}\right)^{2}+\left(\left(\theta_{3}^{h}\right)^{\prime}\right)^{2}}}\left(1,\left(\theta_{2}^{h}\right)^{\prime},\left(\theta_{3}^{h}\right)^{\prime}\right), \\
\boldsymbol{n}^{h} & =\frac{\left(\boldsymbol{t}^{h}\right)^{\prime}}{\left\|\left(\boldsymbol{t}^{h}\right)^{\prime}\right\|} \\
\boldsymbol{b}^{h} & =\boldsymbol{t}^{h} \times \boldsymbol{n}^{h} .
\end{aligned}
$$

We suppose $\boldsymbol{n}^{h} \in C^{1}(0, L)$ which is satisfied if $\left(\theta_{2}^{h}\right)^{\prime \prime},\left(\theta_{3}^{h}\right)^{\prime \prime}$ do not vanish at the same time (which is equivalent to the fact that the curvature of $C^{h}$ is strictly positive for any $\left.x_{1} \in(0, L)\right)$. The case where $C^{h}$ has null curvature points can be treated in the same fashion, provided that we suppose that along these points we have the same degree of smoothness as before with $\boldsymbol{t}^{h}, \boldsymbol{n}^{h}$ and $\boldsymbol{b}^{h}$ appropriately chosen (see Remark 3). We define the map $\boldsymbol{\Theta}^{h}: \bar{\Omega}^{h} \rightarrow \boldsymbol{\Theta}^{h}\left(\bar{\Omega}^{h}\right)=\left\{\hat{\Omega}^{h}\right\}^{-} \subset \mathbb{R}^{3}$, where $\hat{\Omega}^{h}:=\boldsymbol{\Theta}^{h}\left(\Omega^{h}\right)$, in the following manner:

$$
\boldsymbol{\Theta}^{h}\left(x^{h}\right)=\left(x_{1}, \theta_{2}^{h}\left(x_{1}\right), \theta_{3}^{h}\left(x_{1}\right)\right)+x_{2}^{h} \boldsymbol{n}^{h}\left(x_{1}\right)+x_{3}^{h} \boldsymbol{b}^{h}\left(x_{1}\right)
$$

and we assume that $\Theta^{h}$ is a $C^{1}$ diffeomorphism which can be proved if $h$ is small enough and $\theta_{k}^{h}$, for $k=2,3$, are of the form considered here. Namely, we take $\theta_{2}^{h}=h \theta_{2}, \theta_{3}^{h}=h \theta_{3}$ where $\theta_{k} \in C^{3}(0, L)$. Let us suppose

$$
\left(\left(\theta_{1}\right)^{\prime \prime}\right)^{2}\left(x_{1}\right)+\left(\left(\theta_{2}\right)^{\prime \prime}\right)^{2}\left(x_{1}\right) \neq 0
$$

for all $x_{1} \in(0, L)$. A generic point in $\bar{\Omega}^{h}$ or $\left\{\hat{\Omega}^{h}\right\}^{-}$will be denoted by $x^{h}=$ $\left(x_{1}, x_{2}^{h}, x_{3}^{h}\right)$.

Like in $[24,25,12-15,19]$ we start from three dimensional non linear elasticity functional of strain energy (see [7] for an introduction to non linear elasticity)

$$
I^{h}(\boldsymbol{y}):=\frac{1}{h^{2}} \int_{\hat{\Omega}^{h}} W^{h}\left(x^{h}, \nabla \boldsymbol{y}\right) d x^{h}
$$


It is natural to divide the strain energy with $h^{2}$, since the volume is vanishing with the order of $h^{2}$. We are interested in finding $\Gamma$-limit (in some sense i.e. in characterizing the limits of minimizers) of the functionals $\frac{1}{h^{4}} I^{h}$. The reason why we divide with $h^{4}$ is that we want to obtain theory analogous to Föppl-von Kármán for plates and rods (see $[13,14,25]$ ) and Marguerre-von Kármán for shallow shells (see [32]). We do not look the total energy functional because the part with the strain energy contains the highest order derivatives (at least for the external dead loads) and thus makes the most difficult part of the analysis (see Remarks 8 and 10). We shall not impose Dirichlet boundary condition and assume that the body is free at the boundary. The consideration of the other boundary conditions is also possible. We rewrite the functional $I^{h}$ on the domain $\Omega$, i.e. we write

$$
I^{h}(\boldsymbol{y}):=\int_{\Omega} W^{h}\left(\boldsymbol{\Theta}^{h} \circ P^{h}(x),(\nabla \boldsymbol{y}) \circ \boldsymbol{\Theta}^{h} \circ P^{h}\right) \operatorname{det}\left(\left(\nabla \boldsymbol{\Theta}^{h}\right) \circ P^{h}(x)\right) d x,
$$

where by $P^{h}: \mathbb{R}^{3} \rightarrow \mathbb{R}^{3}$ we denote the mapping $P^{h}\left(x_{1}, x_{2}, x_{3}\right)=\left(x_{1}, h x_{2}, h x_{3}\right)$. $(\nabla \boldsymbol{y}) \circ \boldsymbol{\Theta}^{h} \circ P^{h}$ denotes $\nabla \boldsymbol{y}$ evaluated at the point $\boldsymbol{\Theta}^{h}\left(P^{h}(x)\right)$. We assume that for each $h$ it is valid

$$
\operatorname{det}\left(\left(\nabla \boldsymbol{\Theta}^{h}\right) \circ P^{h}(x)\right) W^{h}\left(\boldsymbol{\Theta}^{h} \circ P^{h}(x), \mathbf{F}\right)=W(x, \mathbf{F}), \forall x \in \Omega, \forall \mathbf{F} \in \mathbb{R}^{3 \times 3},
$$

where the stored energy function $W$ is independent of $h$ and satisfies the following assumptions (the same ones as in [25]):

i) $W: \Omega \times \mathbb{R}^{3 \times 3} \rightarrow[0,+\infty]$ is a Carathéodory function; for some $\delta>0$ the function $\mathbf{F} \mapsto W(x, \mathbf{F})$ is of class $C^{2}$ for $\operatorname{dist}(\mathbf{F}, \mathrm{SO}(3))<\delta$ and for a.e. $x \in \Omega$;

ii) the second derivative $\frac{\partial^{2} W}{\partial \mathbf{F}^{2}}$ is a Carathéodory function on the set $\Omega \times\{\mathbf{F} \in$ $\left.\mathbb{R}^{3 \times 3}: \operatorname{dist}(\mathbf{F}, \mathrm{SO}(3))<\delta\right\}$ and there exists a constant $\gamma>0$ such that

$$
\left|\frac{\partial^{2} W}{\partial \mathbf{F}^{2}}(x, \mathbf{F})[\mathbf{G}, \mathbf{G}]\right| \leq \gamma|\mathbf{G}|^{2} \text { if } \operatorname{dist}(\mathbf{F}, \operatorname{SO}(3))<\delta \text { and } \mathbf{G} \in \mathbb{R}_{\mathrm{sym}}^{3 \times 3}
$$

iii) $W$ is frame-indifferent, i.e. $W(x, \mathbf{F})=W(x, \mathbf{R F})$ for a.e. $x \in \Omega$ and every $\mathbf{F} \in \mathbb{R}^{3 \times 3}, \mathbf{R} \in \mathrm{SO}(3)$

iv) $W(x, \mathbf{F})=0$ if $\mathbf{F} \in \mathrm{SO}(3) ; W(x, \mathbf{F}) \geq C \operatorname{dist}^{2}(\mathbf{F}, \mathrm{SO}(3))$ for every $\mathbf{F} \in \mathbb{R}^{3 \times 3}$, where the constant $C>0$ is independent of $x$.

Under these assumptions we first show the compactness result (Theorem 3) i.e. we take the sequence $y^{h} \in W^{1,2}\left(\hat{\Omega}^{h} ; \mathbb{R}^{3}\right)$ such that

$$
\limsup _{h \rightarrow 0} \frac{1}{h^{4}} I^{h}<+\infty
$$

and conclude how that fact affects the limit displacement. In Lemma 2 we prove the lower bound, in Theorem 4 we prove the upper bound and that enables us to identify the limit functional (Theorem 5). First we start with some basic properties of the mappings $\boldsymbol{\Theta}^{h}$ which are necessary for further analysis. 


\section{Properties of the mappings $\Theta^{h}$}

We introduce for $k=2,3$,

$$
p_{k}\left(x_{1}\right)=\frac{\theta_{k}^{\prime \prime}\left(x_{1}\right)}{\sqrt{\left(\theta_{2}^{\prime \prime}\right)^{2}\left(x_{1}\right)+\left(\theta_{3}^{\prime \prime}\right)^{2}\left(x_{1}\right)}} .
$$

Notice that

$$
p_{2}^{2}+p_{3}^{2}=1, p_{2} p_{2}^{\prime}+p_{3} p_{3}^{\prime}=0 .
$$

Let us denote $p=p_{2} p_{3}^{\prime}-p_{2}^{\prime} p_{3}$.

Theorem 1 Let the functions $\theta_{k}^{h}$ be such that

$$
\theta_{k}^{h}\left(x_{1}\right)=h \theta_{k}\left(x_{1}\right), \text { for all } x_{1} \in(0, L), k=2,3 .
$$

where $\theta_{k} \in C^{3}(0, L)$ is independent of $h$. Then there exists $h_{0}=h_{0}(\theta)>0$ such that the Jacobian matrix $\nabla \boldsymbol{\Theta}^{h}\left(x^{h}\right)$, where the mappings $\boldsymbol{\Theta}^{h}$ are defined with (2.8), is invertible for all $x^{h} \in \bar{\Omega}^{h}$ and all $h \leq h_{0}$. Also there exists $C>0$ such that for $h \leq h_{0}$ we have

$$
\operatorname{det} \nabla \Theta^{h}=1+h \delta^{h}\left(x^{h}\right),
$$

and

$$
\begin{aligned}
\boldsymbol{t}^{h}\left(x_{1}\right)= & \boldsymbol{e}_{1}+h \theta_{2}^{\prime}\left(x_{1}\right) \boldsymbol{e}_{2}+h \theta_{3}^{\prime}\left(x_{1}\right) \boldsymbol{e}_{3}+h^{2} \boldsymbol{o}_{1}\left(x_{1}\right), \\
\boldsymbol{n}^{h}\left(x_{1}\right)= & p_{2}\left(x_{1}\right) \boldsymbol{e}_{2}+p_{3}\left(x_{1}\right) \boldsymbol{e}_{3}-h\left(\theta_{2}^{\prime} p_{2}+\theta_{3}^{\prime} p_{3}\right)\left(x_{1}\right) \boldsymbol{e}_{1} \\
& +h^{2} \boldsymbol{o}_{2}\left(x_{1}\right), \\
\boldsymbol{b}^{h}\left(x_{1}\right)= & -p_{3}\left(x_{1}\right) \boldsymbol{e}_{2}+p_{2}\left(x_{1}\right) \boldsymbol{e}_{3}+h\left(\theta_{2}^{\prime} p_{3}-\theta_{3}^{\prime} p_{2}\right)\left(x_{1}\right) \boldsymbol{e}_{1} \\
& +h^{2} \boldsymbol{o}_{3}\left(x_{1}\right), \\
\nabla \boldsymbol{\Theta}^{h}\left(x^{h}\right)= & \mathbf{R}_{e}\left(x_{1}\right)+h \mathbf{C}\left(x_{1}\right)+x_{2}^{h} \mathbf{D}\left(x_{1}\right)+x_{3}^{h} \mathbf{E}\left(x_{1}\right) \\
& +h^{2} \mathbf{O}_{1}^{h}\left(x^{h}\right), \\
\left(\nabla \boldsymbol{\Theta}^{h}\left(x^{h}\right)\right)^{-1}= & \mathbf{R}_{e}^{T}\left(x_{1}\right)-h \mathbf{C}_{1}\left(x_{1}\right)-x_{2}^{h} \mathbf{D}_{1}\left(x_{1}\right)-x_{3}^{h} \mathbf{E}_{1}\left(x_{1}\right) \\
& +h^{2} \mathbf{O}_{2}^{h}\left(x^{h}\right), \\
\| & \left(\nabla \boldsymbol{\Theta}^{h}\right)-\mathbf{R}_{e} \|_{L^{\infty}\left(\Omega^{h} ; \mathbb{R}^{3 \times 3}\right)}<C h, \\
\| & \left(\nabla \boldsymbol{\Theta}^{h}\right)^{-1}-\mathbf{R}_{e}^{T} \|_{L^{\infty}\left(\Omega^{h} ; \mathbb{R}^{3 \times 3}\right)}<C h,
\end{aligned}
$$

where

$$
\begin{aligned}
\mathbf{R}_{e} & =\left(\begin{array}{c|c}
1 & \mathbf{0} \\
\hline \mathbf{0} & \mathbf{R}
\end{array}\right), \mathbf{R}=\left(\begin{array}{cc}
p_{2} & -p_{3} \\
p_{3} & p_{2}
\end{array}\right) \\
\mathbf{C} & =\left(\begin{array}{ccc}
0 & -\left(\theta_{2}^{\prime} p_{2}+\theta_{3}^{\prime} p_{3}\right)-\left(\theta_{3}^{\prime} p_{2}-\theta_{2}^{\prime} p_{3}\right) \\
\theta_{2}^{\prime} & 0 & 0 \\
\theta_{3}^{\prime} & 0 & 0
\end{array}\right) \\
\mathbf{D} & =\left(\begin{array}{lll}
0 & 0 & 0 \\
p_{2}^{\prime} & 0 & 0 \\
p_{3}^{\prime} & 0 & 0
\end{array}\right), \mathbf{E}=\left(\begin{array}{ccc}
0 & 0 & 0 \\
-p_{3}^{\prime} & 0 & 0 \\
p_{2}^{\prime} & 0 & 0
\end{array}\right)
\end{aligned}
$$




$$
\begin{aligned}
\mathbf{C}_{1} & =\left(\begin{array}{ccc}
0 & -\theta_{2}^{\prime} & -\theta_{3}^{\prime} \\
\theta_{2}^{\prime} p_{2}+\theta_{3}^{\prime} p_{3} & 0 & 0 \\
\theta_{3}^{\prime} p_{2}-\theta_{2}^{\prime} p_{3} & 0 & 0
\end{array}\right), \\
\mathbf{D}_{1} & =\left(\begin{array}{lll}
0 & 0 & 0 \\
0 & 0 & 0 \\
p & 0 & 0
\end{array}\right), \mathbf{E}_{1}=\left(\begin{array}{ccc}
0 & 0 & 0 \\
-p & 0 & 0 \\
0 & 0 & 0
\end{array}\right),
\end{aligned}
$$

and $\delta^{h}: \bar{\Omega}^{h} \rightarrow \mathbb{R}, \boldsymbol{o}_{i}:(0, L) \rightarrow \mathbb{R}^{3}, i=1,2,3, \mathbf{O}_{k}^{h}: \bar{\Omega}^{h} \rightarrow \mathbb{R}^{3 \times 3}, k=1,2$ are functions which satisfy

$$
\begin{gathered}
\sup _{0<h \leq h_{0} x^{h} \in \bar{\Omega}^{h}}\left|\delta^{h}\left(x^{h}\right)\right| \leq C_{0}, \\
\sup _{0<h \leq h_{0}} \max _{x_{1} \in(0, L)}\left\|\boldsymbol{o}_{i}^{h}\left(x_{1}\right)\right\| \leq C_{0}, \sup _{0<h \leq h_{0}} \max _{x_{1} \in(0, L)}\left\|\left(\boldsymbol{o}_{i}^{h}\right)^{\prime}\left(x_{1}\right)\right\| \leq C_{0} \\
\sup _{0<h \leq h_{0}} \max _{i, j} \max _{x^{h} \in \bar{\Omega}^{h}}\left\|\mathbf{O}_{k, i j}^{h}\left(x^{h}\right)\right\| \leq C_{0}, \quad k=1,2,
\end{gathered}
$$

for some constant $C_{0}>0$.

Proof. It can be easily seen that

$$
\boldsymbol{t}^{h}\left(x_{1}\right)=\boldsymbol{e}_{1}+h \theta_{2}^{\prime}\left(x_{1}\right) \boldsymbol{e}_{2}+h \theta_{3}^{\prime}\left(x_{1}\right) \boldsymbol{e}_{3}-\frac{h^{2}}{2}\left(\left(\theta_{2}^{\prime}\right)^{2}+\left(\theta_{3}^{\prime}\right)^{2}\right) \boldsymbol{e}_{1}+h^{3} \boldsymbol{o}_{4}^{h}\left(x^{h}\right),
$$

where $\left\|\boldsymbol{o}_{4}\right\|_{C^{2}(0, L)} \leq C$. The relations (3.5) and (3.6) are the direct consequences of the relation (3.16). Let us by $\boldsymbol{u}^{h}:(0, L) \rightarrow \mathbb{R}^{3}$ denote the function

$$
\boldsymbol{u}^{h}=\left(1, h \theta_{2}^{\prime}, h \theta_{3}^{\prime}\right)^{T}
$$

It is easy to see that

$$
\nabla \boldsymbol{\Theta}^{h}\left(x^{h}\right)=\left(\boldsymbol{u}^{h}\left(x_{1}\right)+x_{2}^{h}\left(\boldsymbol{n}^{h}\right)^{\prime}\left(x_{1}\right)+x_{3}^{h}\left(\boldsymbol{b}^{h}\right)^{\prime}\left(x_{1}\right)\left|\boldsymbol{n}^{h}\left(x_{1}\right)\right| \boldsymbol{b}^{h}\left(x_{1}\right)\right) .
$$

The relations (3.3), (3.7), (3.9), (3.10) are the direct consequences of the relations (3.4-(3.6) and (3.18). The relation (3.8) is the direct consequence of the fact that, for a regular matrix $\mathbf{A}$ and arbitrary $\mathbf{B}$, which satisfies $\left\|\mathbf{A}^{-1} \mathbf{B}\right\|<1(\|\cdot\|$ is the operational norm), the matrix $\mathbf{A}+\mathbf{B}$ is invertible and

$$
\left\|(\mathbf{A}+\mathbf{B})^{-1}-\left(\mathbf{A}^{-1}-\mathbf{A}^{-1} \mathbf{B} \mathbf{A}^{-1}\right)\right\| \leq \frac{\left\|\mathbf{A}^{-1} \mathbf{B}\right\|^{2}\|\mathbf{A}\|^{-1}}{1-\left\|\mathbf{A}^{-1} \mathbf{B}\right\|} .
$$

To end the proof observe that

$$
\mathbf{C}_{1}=\mathbf{R}_{e}^{T} \mathbf{C R}_{e}^{T}, \mathbf{D}_{1}=\mathbf{R}_{e}^{T} \mathbf{D} \mathbf{R}_{e}^{T}, \mathbf{E}_{1}=\mathbf{R}_{e}^{T} \mathbf{E} \mathbf{R}_{e}^{T} .
$$

Remark 1 By a careful computation it can be seen that $\boldsymbol{o}_{2}^{h}, \boldsymbol{o}_{3}^{h}$ and $\boldsymbol{o}_{4}^{h}$ (defined in the relation (3.16)) are dominantly in $\boldsymbol{e}_{2}, \boldsymbol{e}_{3}$ plane i.e. that we have for $i=2,3,4$ :

$$
\boldsymbol{o}_{i}^{h}\left(x_{1}\right)=f_{i}^{2}\left(x_{1}\right) \boldsymbol{e}_{2}+f_{i}^{3}\left(x_{1}\right) \boldsymbol{e}_{3}+h \boldsymbol{r}_{i}^{h}\left(x_{1}\right),
$$

where $f_{i}^{2}, f_{i}^{3} \in C^{1}(0, L), \sup _{0<h \leq h_{0}}\left\|\boldsymbol{r}_{i}^{h}\right\|_{C^{1}(0, L)} \leq C$, for some $C>0$. 
Remark 2 By a further inspection it can be seen that

$$
\begin{aligned}
& f_{4}^{2}=-\frac{1}{2} \theta_{2}^{\prime}\left(\left(\theta_{2}^{\prime}\right)^{2}+\left(\theta_{3}^{\prime}\right)^{2}\right), f_{4}^{3}=-\frac{1}{2} \theta_{3}^{\prime}\left(\left(\theta_{2}^{\prime}\right)^{2}+\left(\theta_{3}^{\prime}\right)^{2}\right), \\
& f_{2}^{2}=p_{2}\left(f_{2}\left(\theta^{\prime}, \theta^{\prime \prime}\right)-\frac{1}{2}\left(\left(\theta_{2}^{\prime}\right)^{2}+\left(\theta_{3}^{\prime}\right)^{2}\right)\right)-\theta_{2}^{\prime}\left(\theta_{2}^{\prime} p_{2}+\theta_{3}^{\prime} p_{3}\right), \\
& f_{2}^{3}=p_{3}\left(f_{2}\left(\theta^{\prime}, \theta^{\prime \prime}\right)-\frac{1}{2}\left(\left(\theta_{2}^{\prime}\right)^{2}+\left(\theta_{3}^{\prime}\right)^{2}\right)\right)-\theta_{3}^{\prime}\left(\theta_{2}^{\prime} p_{2}+\theta_{3}^{\prime} p_{3}\right), \\
& f_{3}^{2}=p_{3}\left(\left(\theta_{2}^{\prime}\right)^{2}+\left(\theta_{3}^{\prime}\right)^{2}\right)-p_{3} f_{2}\left(\theta^{\prime}, \theta^{\prime \prime}\right), \\
& f_{3}^{3}=-p_{2}\left(\left(\theta_{2}^{\prime}\right)^{2}+\left(\theta_{3}^{\prime}\right)^{2}\right)+p_{2} f_{2}\left(\theta^{\prime}, \theta^{\prime \prime}\right),
\end{aligned}
$$

where $f_{2}\left(\theta^{\prime}, \theta^{\prime \prime}\right) \in C^{1}(0, L)$ is the expression that includes $\theta^{\prime}, \theta^{\prime \prime}$ :

$$
\begin{aligned}
f_{2}\left(\theta^{\prime}, \theta^{\prime \prime}\right)= & \frac{1}{2}\left(\left(p_{2}+p_{3}\right)\left(\left(\theta_{2}^{\prime}\right)^{2}+\left(\theta_{3}^{\prime}\right)^{2}\right)+2\left(\theta_{2}^{\prime}+\theta_{3}^{\prime}\right)\left(\theta_{2}^{\prime} p_{2}+\theta_{3}^{\prime} p_{3}\right)\right. \\
& \left.-\sqrt{\left(\theta_{2}^{\prime \prime}\right)^{2}+\left(\theta_{3}^{\prime \prime}\right)^{2}}\left(\theta_{2}^{\prime} p_{2}+\theta_{3}^{\prime} p_{3}\right)^{2}\right) .
\end{aligned}
$$

Remark 3 It is not necessary to impose the condition (2.9). All we need is the existence of the expansions given by (3.4)-(3.6), where $p_{2}, p_{3} \in C^{1}(0, L)$, including the statement of Remark 1.

Remark 4 Although $\boldsymbol{\Theta}^{h}$ makes the small perturbation of the central line, $\left(x_{1}, 0,0\right)$, for $x_{1} \in[0, L]$, it is not true that $\nabla \Theta^{h}$ is close to the identity (like in the shallow shell model, see [32]). In fact, there are torsional effects of order 0 on every cross section. This is the main reason why is the change of coordinates introduced in the next chapter useful.

\section{$4 \Gamma$-convergence}

We shall need the following theorem which can be found in [12].

Theorem 2 (on geometric rigidity) Let $U \subset \mathbb{R}^{m}$ be a bounded Lipschitz domain, $m \geq 2$. Then there exists a constant $C(U)$ with the following property: for every $\boldsymbol{v} \in$ $W^{1,2}\left(U ; \mathbb{R}^{m}\right)$ there is an associated rotation $\mathbf{R} \in \mathrm{SO}(m)$ such that

$$
\|\nabla \boldsymbol{v}-\mathbf{R}\|_{L^{2}(U)} \leq C(U) \| \operatorname{dist}\left(\nabla \boldsymbol{v}, \mathrm{SO}(m) \|_{L^{2}(U)} .\right.
$$

The constant $C(U)$ can be chosen uniformly for a family of domains which are Bilipschitz equivalent with controlled Lipschitz constants. The constant $C(U)$ is invariant under dilatations.

The following version of the Korn's inequality is needed.

Lemma 1 Let $\omega \subset \mathbb{R}^{2}$ with Lipschitz boundary and $\boldsymbol{u} \in L^{2}\left(\omega ; \mathbb{R}^{2}\right)$. Let us by $e_{i j}(u)$ denote $e_{i j}(u)=\frac{1}{2}\left(\partial_{i} \boldsymbol{u}+\partial_{j} \boldsymbol{u}\right)$. Let us suppose that for every $i, j=1,2$ we have that $e_{i j}(u) \in L^{2}(\omega)$. Then we have that $\boldsymbol{u} \in W^{1,2}\left(\omega ; \mathbb{R}^{2}\right)$. Also there exists constant $C(\omega)$, depending only on the domain $\omega$, such that we have

$$
\begin{aligned}
\|\boldsymbol{u}\|_{W^{1,2}\left(\omega ; \mathbb{R}^{2}\right)} \leq & C(\omega)\left(\left|\int_{\omega} \boldsymbol{u} d x_{1} d x_{2}\right|+\left|\int_{\omega}\left(x_{1} \boldsymbol{u}_{2}-x_{2} \boldsymbol{u}_{1}\right) d x_{1} d x_{2}\right|\right. \\
& \left.+\sum_{i, j=1,2}\left\|e_{i j}(\boldsymbol{u})\right\|_{L^{2}(\omega)}\right) .
\end{aligned}
$$


Let us suppose that the domains $\omega_{s}$ are changing in the sense that they are equal to $\omega_{s}=\mathbf{A}_{s} \omega$, where $\mathbf{A}_{s} \in \mathbb{R}^{2 \times 2}$, and there exists a constant $C$ such that $\left\|\mathbf{A}_{s}\right\|,\left\|\mathbf{A}_{s}^{-1}\right\| \leq$ $C$. Then the constant in the inequality (4.2) can be chosen independently of $s$.

Proof. The first part of the lemma (the fixed domain) is a version of the Korn's inequality (see e.g. [29]). The last part we shall prove by a contradiction. Let us suppose the contrary that for each $n \in \mathbf{N}$ there exists $s^{n}$ and $\boldsymbol{u}^{n} \in W^{1,2}\left(\omega_{s^{n}} ; \mathbb{R}^{2}\right)$ such that we have

$$
\begin{aligned}
& \left|\int_{\omega_{s^{n}}} \boldsymbol{u}^{n} d x_{1} d x_{2}\right|+\left|\int_{\omega_{s} n}\left(x_{1} \boldsymbol{u}_{2}^{n}-x_{2} \boldsymbol{u}_{1}^{n}\right) d x_{1} d x_{2}\right| \\
& +\sum_{i, j=1,2}\left\|e_{i j}^{s^{n}}\left(\boldsymbol{u}^{n}\right)\right\|_{L^{2}\left(\omega_{s} n\right)} \leq \frac{1}{n}\left\|\boldsymbol{u}^{n}\right\|_{W^{1,2}\left(\omega_{s} n ; \mathbb{R}^{2}\right)},
\end{aligned}
$$

where we have by $e_{i j}^{s^{n}}(\cdot)$ denoted the symmetrized gradient on the domain $\omega_{s^{n}}$. Without any loss of generality we can suppose that $\left\|\boldsymbol{u}^{n}\right\|_{W^{1,2}\left(\omega_{s}^{n} ; \mathbb{R}^{2}\right)}=1$. Let us take the subsequence of $\left(s^{n}\right)$ (still denoted by $\left(s^{n}\right)$ ) such that $\mathbf{A}_{s^{n}} \rightarrow \mathbf{A}$ and $\mathbf{A}_{s^{n}}^{-1} \rightarrow \mathbf{A}^{-1}$ in $\mathbb{R}^{2 \times 2}$.

Let us look the sequence $\boldsymbol{u}_{c}^{n}=\boldsymbol{u}^{n} \circ \mathbf{A}_{s^{n}} \circ \mathbf{A}^{-1}$. It is clear that there exist $C_{1}, C_{2}>0$ such that

$$
C_{1} \leq\left\|\boldsymbol{u}_{c}^{n}\right\|_{W^{1,2}\left(\omega_{\infty} ; \mathbb{R}^{2}\right)} \leq C_{2}
$$

where we have put $\omega_{\infty}:=\mathbf{A} \omega$. Thus there exists $\boldsymbol{u} \in W^{1,2}\left(\omega_{\infty} ; \mathbb{R}^{2}\right)$ such that $\boldsymbol{u}_{c}^{n} \rightarrow \boldsymbol{u}$ weakly in $W^{1,2}\left(\omega_{\infty} ; \mathbb{R}^{2}\right)$. Specially, by the compactness of the embedding $L^{2} \hookrightarrow W^{1,2}$ (see e.g. [2]), we also conclude the strong convergence $\boldsymbol{u}_{c}^{n} \rightarrow \boldsymbol{u}$ in $L^{2}\left(\omega_{\infty} ; \mathbb{R}^{2}\right)$. Since it is valid $\mathbf{A}_{s^{n}} \mathbf{A}^{-1} \rightarrow \mathbf{I}$, it can be easily seen that, from the weak convergence, it follows $e_{i j}^{s^{n}}\left(\boldsymbol{u}^{n}\right) \circ\left(\mathbf{A}_{s^{n} \circ \mathbf{A}^{-1}}\right) \rightarrow e_{i j}^{\infty}(\boldsymbol{u})$, weakly in $L^{2}\left(\omega_{\infty} ; \mathbb{R}^{2}\right)$, where we have by $e_{i j}^{\infty}(\cdot)$ denoted the symmetrized gradient on the domain $\omega_{\infty}$. From the weak convergence we can conclude that

$$
\left\|e_{i j}^{\infty}(\boldsymbol{u})\right\|_{L^{2}\left(\omega_{\infty}\right)} \leq \liminf _{n \rightarrow \infty}\left\|e_{i j}^{s^{n}}\left(\boldsymbol{u}^{n}\right) \circ\left(\mathbf{A}_{s^{n}} \circ \mathbf{A}^{-1}\right)\right\|_{L^{2}\left(\omega_{\infty}\right)}=0
$$

for every $i, j=1,2$. We can also from (4.3) conclude that

$$
\int_{\omega_{\infty}} \boldsymbol{u} d x_{1} d x_{2}=0, \int_{\omega_{\infty}}\left(x_{1} \boldsymbol{u}_{2}-x_{2} \boldsymbol{u}_{1}\right) d x_{1} d x_{2}=0 .
$$

Applying the standard Korn's inequality on the domain $\omega_{\infty}$, i.e.

$$
\begin{aligned}
\left\|\boldsymbol{u}-\boldsymbol{u}_{c}^{n}\right\|_{W^{1,2}\left(\omega_{\infty} ; \mathbb{R}^{2}\right) \leq} & C\left(\omega_{\infty}\right)\left(\left\|\boldsymbol{u}-\boldsymbol{u}_{c}^{n}\right\|_{L^{2}\left(\omega_{\infty} ; \mathbb{R}^{2}\right)}\right. \\
& \left.+\sum_{i, j=1,2}\left\|e_{i j}(\boldsymbol{u})-e_{i j}\left(\boldsymbol{u}_{c}^{n}\right)\right\|_{L^{2}\left(\omega_{\infty} ; \mathbb{R}^{2}\right)}\right),
\end{aligned}
$$

we conclude that $\boldsymbol{u}_{c}^{n} \rightarrow \boldsymbol{u}$ strongly in $W^{1,2}\left(\omega_{\infty} ; \mathbb{R}^{2}\right)$. But then (4.4), (4.5), (4.6) make a contradiction with the version of the Korn's inequality (4.2) on the domain $\omega_{\infty}$

Remark 5 The same proof can be done under the assumption that $\omega_{s}=F_{s}(\omega)$, where $F_{s}$ is the family of Bilipschitz mappings whose Bilipschitz constants we can control (i.e. the Lipschitz constants of $F_{s}$ and $F_{s}^{-1}$ are bounded by a universal constant), provided that the family $F_{s}$ is strongly compact in $W^{1, \infty}\left(\omega ; \mathbb{R}^{2}\right)$. It would require more analysis to conclude the same result only for Bilipschitz mappings whose Bilipschitz constants we can control. 
Let us by $\boldsymbol{x}^{\prime}: \mathbb{R}^{3} \rightarrow \mathbb{R}^{3}$ denote the change of coordinates

$$
\left(x_{1}^{\prime}, x_{2}^{\prime}, x_{3}^{\prime}\right)=\boldsymbol{x}^{\prime}\left(x_{1}, x_{2}, x_{3}\right):=\mathbf{R}_{e}\left(x_{1}\right)\left(\begin{array}{l}
x_{1} \\
x_{2} \\
x_{3}
\end{array}\right) .
$$

By $\Omega^{\prime}$ we denote $\boldsymbol{x}^{\prime}(\Omega)$ and $\omega^{\prime}\left(x_{1}\right) \subset \mathbb{R}^{2}$ denotes $\boldsymbol{x}^{\prime}\left(\left\{x_{1}\right\} \times \omega\right)$, for $x_{1} \in[0, L]$. The generic point in $\Omega^{\prime}$ is denoted with $x^{\prime}=\left(x_{1}, x_{2}^{\prime}, x_{3}^{\prime}\right)$. Let us observe that by $(2.2)$ and (2.3)

$$
\begin{aligned}
& \int_{\omega^{\prime}\left(x_{1}\right)} x_{2}^{\prime} d x_{2}^{\prime} d x_{3}^{\prime}=\int_{\omega^{\prime}\left(x_{1}\right)} x_{3}^{\prime} d x_{2}^{\prime} d x_{3}^{\prime}=0, \\
& \int_{\omega^{\prime}\left(x_{1}\right)} x_{2}^{\prime} x_{3}^{\prime} d x_{2}^{\prime} d x_{3}^{\prime}=p_{2} p_{3} \int_{\omega}\left(x_{2}^{2}-x_{3}^{2}\right) d x_{2} d x_{3}, \\
& \mu(\omega)=\int_{\omega}\left(x_{2}^{2}+x_{3}^{2}\right) d x_{2} d x_{3}=\int_{\omega^{\prime}\left(x_{1}\right)}\left(\left(x_{2}^{\prime}\right)^{2}+\left(x_{3}^{\prime}\right)^{2}\right) d x_{2}^{\prime} d x_{3}^{\prime},
\end{aligned}
$$

for all $x_{1} \in[0, L]$. By $\left.\left(\partial_{i} \boldsymbol{y}_{j}\right) \circ \boldsymbol{\Theta}^{h} \circ P^{h}\right)$ we denote $\partial_{i} \boldsymbol{y}_{j}$ evaluated at the point $\Theta^{h}\left(P^{h}(x)\right)$.

In the sequel we suppose $h_{0} \geq 1$ (see Theorem 1 ). If this was not the case, what follows could be easily adapted. Using theorem 2 we can prove the following theorem

Theorem 3 Let $\boldsymbol{y}^{h} \in W^{1,2}\left(\hat{\Omega}^{h} ; \mathbb{R}^{3}\right)$ and let

$$
E^{h}=\frac{1}{h^{2}} \int_{\hat{\Omega}^{h}} \operatorname{dist}^{2}\left(\nabla \boldsymbol{y}^{h}, \mathrm{SO}(3)\right) d x .
$$

Let us suppose that

$$
\limsup _{h \rightarrow 0} \frac{E^{h}}{h^{4}}<+\infty .
$$

Then there exist maps $\mathbf{R}^{h}:[0, L] \rightarrow \mathrm{SO}(3)$ and $\widetilde{\mathbf{R}}^{h}:[0, L] \rightarrow \mathbb{R}^{3 \times 3}$, with $|\widetilde{\mathbf{R}}| \leq C$, $\widetilde{\mathbf{R}} \in W^{1,2}\left([0, L], \mathbb{R}^{3 \times 3}\right)$ and constants $\overline{\mathbf{R}}^{h} \in \mathrm{SO}(3), c^{h} \in \mathbb{R}^{3}$ such that the functions $\widetilde{\boldsymbol{y}}^{h}:=\left(\overline{\mathbf{R}}^{h}\right)^{T} \boldsymbol{y}^{h}-c^{h}$ satisfy

$$
\begin{gathered}
\left\|\left(\nabla \widetilde{\boldsymbol{y}}^{h}\right) \circ \boldsymbol{\Theta}^{h} \circ P^{h}-\mathbf{R}^{h}\right\|_{L^{2}(\Omega)} \leq C h^{2}, \\
\left\|\mathbf{R}^{h}-\widetilde{\mathbf{R}}^{h}\right\|_{L^{2}([0, L])} \leq C h^{2}, \quad\left\|\left(\widetilde{\mathbf{R}}^{h}\right)^{\prime}\right\|_{L^{2}([0, L])} \leq C h, \\
\left\|\mathbf{R}^{h}-\mathbf{I}\right\|_{L^{\infty}([0, L])} \leq C h .
\end{gathered}
$$

Moreover if we define

$$
\begin{aligned}
u^{h} & =\frac{1}{A} \int_{\omega} \frac{\widetilde{\boldsymbol{y}}_{1}^{h} \circ \boldsymbol{\Theta}^{h} \circ P^{h}-x_{1}}{h^{2}} d x_{2} d x_{3}, \\
v_{k}^{h} & =\frac{1}{A} \int_{\omega} \frac{\widetilde{\boldsymbol{y}}_{k}^{h} \circ \boldsymbol{\Theta}^{h} \circ P^{h}-h \theta_{k}}{h} d x_{2} d x_{3}, \text { for } k=2,3, \\
w^{h} & =\frac{1}{A \mu(\omega)} \int_{\omega} \frac{x_{2}^{\prime}\left(\widetilde{\boldsymbol{y}}_{3} \circ \boldsymbol{\Theta}^{h} \circ P^{h}\right)-x_{3}^{\prime}\left(\widetilde{\boldsymbol{y}}_{2} \circ \boldsymbol{\Theta}^{h} \circ P^{h}\right)}{h^{2}} d x_{2} d x_{3}
\end{aligned}
$$

then, up to subsequences, the following properties are satisfied 
(a) $u^{h} \rightarrow u$ in $W^{1,2}(0, L)$;

(b) $v_{k}^{h} \rightarrow v_{k}$ in $W^{1,2}(0, L)$, where $v_{k} \in W^{2,2}(0, L)$ for $k=2,3$.

(c) $w^{h} \rightarrow w$ weakly in $W^{1,2}(0, L)$;

(d) $\frac{\left(\nabla \widetilde{\boldsymbol{y}}^{h}\right) \circ \boldsymbol{\Theta}^{h} \circ P^{h}-\mathbf{I}}{h} \rightarrow \mathbf{A}$, in $L^{2}(\Omega)$, where $\mathbf{A} \in W^{1,2}(0, L)$ is given by

$$
\mathbf{A}=\left(\begin{array}{ccc}
0 & -v_{2}^{\prime} & -v_{3}^{\prime} \\
v_{2}^{\prime} & 0 & -w \\
v_{3}^{\prime} & w & 0
\end{array}\right)
$$

(e) $\operatorname{sym} \frac{\mathbf{R}^{h}-\mathbf{I}}{h^{2}} \rightarrow \frac{\mathbf{A}^{2}}{2}$ uniformly on $(0, L)$;

(f) the sequence $\boldsymbol{\gamma}^{h}$ defined by

$$
\begin{aligned}
\gamma_{1}^{h}(x)=\frac{1}{h}( & \frac{\left(\widetilde{\boldsymbol{y}}_{1}^{h} \circ \boldsymbol{\Theta}^{h} \circ P^{h}\right)(x)-x_{1}}{h^{2}}-u^{h}\left(x_{1}\right) \\
& \left.+x_{2}^{\prime}\left(\left(v_{2}^{h}\right)^{\prime}+\theta_{2}^{\prime}\right)\left(x_{1}\right)+x_{3}^{\prime}\left(\left(v_{3}^{h}\right)^{\prime}+\theta_{3}^{\prime}\right)\left(x_{1}\right)\right), \\
\gamma_{k}^{h}(x)=\frac{1}{h^{2}}( & \frac{\left(\widetilde{\boldsymbol{y}}_{k}^{h} \circ \boldsymbol{\Theta}^{h} \circ P^{h}\right)(x)-h \theta_{k}-h x_{k}^{\prime}}{h} \\
& \left.-v_{k}^{h}\left(x_{1}\right)-h\left(x_{k}^{\prime}\right)^{\perp} \omega^{h}\left(x_{1}\right)\right), \text { for } k=2,3,
\end{aligned}
$$

where $\left(x^{\prime}\right)^{\perp}:=\left(0,-x_{3}^{\prime}, x_{2}^{\prime}\right)$, is weakly convergent in $L^{2}(\Omega)$ to a function $\boldsymbol{\gamma}$ belonging to the space $\mathcal{C}$, where

$$
\begin{aligned}
& \mathcal{C}=\left\{\gamma \in L^{2}\left(\Omega ; \mathbb{R}^{3}\right): \int_{\omega} \gamma=0, \partial_{2} \gamma, \partial_{3} \gamma \in L^{2}\left(\Omega ; \mathbb{R}^{3}\right),\right. \\
& \left.\int_{\omega}\left(x_{3}^{\prime} \gamma_{2}\left(x_{1}, \cdot\right)-x_{2}^{\prime} \gamma_{3}\left(x_{1}, \cdot\right)\right) d x_{2} d x_{3}=0, \text { for a.e. } x_{1} \in(0, L)\right\} .
\end{aligned}
$$

Moreover $\partial_{k} \gamma^{h} \rightarrow \partial_{k} \gamma$ in $L^{2}(\Omega)$ for $k=2,3$,

Proof. We follow the proof of Theorem 2.2 in [25]. Applying Theorem 2 as in the compactness result of [24] (using the boundedness of $\nabla \boldsymbol{\Theta}^{h}$ and $\left(\nabla \boldsymbol{\Theta}^{h}\right)^{-1}$ we can find a sequence of piecewise constant maps $\mathbf{R}^{h}:[0, L] \rightarrow \mathrm{SO}(3)$ such that

$$
\int_{\Omega}\left\|\left(\nabla \boldsymbol{y}^{h}\right) \circ \boldsymbol{\Theta}^{h} \circ P^{h}-\mathbf{R}^{h}\right\|^{2} d x \leq C h^{4},
$$

and

$$
\int_{I^{\prime}}\left\|\mathbf{R}^{h}\left(x_{1}+\xi\right)-\mathbf{R}\left(x_{1}\right)\right\|^{2} d x_{1} \leq C h^{2}(|\xi|+h)^{2},
$$

where $I^{\prime}$ is any open interval in $(0, L)$ and $\xi \in \mathbb{R}$ satisfies $|\xi| \leq \operatorname{dist}\left(I^{\prime},\{0, L\}\right)$. Let $\eta \in C_{0}^{\infty}(0,1)$ be such that $\eta \geq 0$ and $\int_{0}^{1} \eta(s) d s=1$. We set $\eta_{h}=\frac{1}{h} \eta\left(\frac{s}{h}\right)$ and we define

$$
\widetilde{\mathbf{R}}^{h}\left(x_{1}\right):=\int_{-h}^{h} \eta_{h}(s) \mathbf{R}^{h}\left(x_{1}-s\right) d s
$$


where we have extended $\mathbf{R}^{h}$ outside $[0, L]$ by taking $\mathbf{R}^{h}\left(x_{1}\right)=\mathbf{R}^{h}(0)$ for every $x_{1}<0, \mathbf{R}^{h}\left(x_{1}\right)=\mathbf{R}^{h}(L)$ for every $x_{1}>L$. Clearly $\left\|\widetilde{\mathbf{R}}^{h}\right\| \leq C$ for every $h$ while properties (4.13) follow from properties (4.21). Moreover since by construction (see $[24])$

$$
\left\|\mathbf{R}^{h}\left(x_{1}+s\right)-\mathbf{R}^{h}\left(x_{1}\right)\right\|^{2} \leq \frac{C}{h^{3}} \int_{\hat{\Omega}^{h}} \operatorname{dist}^{2}\left(\nabla \boldsymbol{y}^{h}, \mathrm{SO}(3)\right) \leq C h^{3},
$$

for every $|s| \leq h$ we have by Jensen's inequality that

$$
\left\|\widetilde{\mathbf{R}}^{h}-\mathbf{R}^{h}\right\|_{L^{\infty}\left([0, L] ; \mathbb{R}^{3 \times 3}\right.}^{2} \leq C h^{3}
$$

By the Sobolev-Poincare inequality and the second inequality in (4.13), there exist constants $\mathbf{Q}^{h} \in \mathbb{R}^{3 \times 3}$ such that $\left\|\widetilde{\mathbf{R}}^{h}-\mathbf{Q}^{h}\right\|_{L^{\infty}\left([0, L] ; \mathbb{R}^{3 \times 3}\right)} \leq C h$. Combining this inequality with (4.22), we have that $\left\|\mathbf{R}^{h}-\mathbf{Q}^{h}\right\|_{L^{\infty}\left([0, L] ; \mathbb{R}^{3 \times 3}\right)} \leq C h$. This implies that $\operatorname{dist}\left(\mathbf{Q}^{h}, \mathrm{SO}(3)\right) \leq C h$; thus, we may assume that $\mathbf{Q}^{h}$ belongs to $\mathrm{SO}(3)$ and by modifying $\mathbf{Q}^{h}$ by order $h$, if needed. Now choosing $\overline{\mathbf{R}}^{h}=\mathbf{Q}^{h}$ and replacing $\mathbf{R}^{h}$ by $\left(\mathbf{Q}^{h}\right)^{T} \mathbf{R}^{h}$ and $\widetilde{\mathbf{R}}^{h}$ by $\left(\mathbf{Q}^{h}\right)^{T} \widetilde{\mathbf{R}}^{h}$, we obtain (4.14). By suitable choice of constants $c^{h} \in \mathbb{R}^{3}$ we may assume that

$$
\int_{\Omega}\left(\widetilde{\boldsymbol{y}}_{1}^{h} \circ \boldsymbol{\Theta}^{h} \circ P^{h}-x_{1}\right)=0, \quad \int_{\Omega}\left(\widetilde{\boldsymbol{y}}_{k}^{h} \circ \boldsymbol{\Theta}^{h} \circ P^{h}-h \theta_{k}\right)=0, \text { for } k=2,3 .
$$

Let $\mathbf{A}^{h}=\frac{\mathbf{R}^{h}-\mathbf{I}}{h}$. By (4.14) there exists $\mathbf{A} \in L^{\infty}\left((0, L) ; \mathbb{R}^{3 \times 3}\right)$ such that, up to subsequences,

$$
\mathbf{A}^{h} \rightarrow \mathbf{A} \text { weakly } * \text { in } L^{\infty}\left((0, L) ; \mathbb{R}^{3 \times 3}\right) .
$$

On the other hand it follows from (4.13) and (4.14) that

$$
\frac{\widetilde{\mathbf{R}}^{h}-\mathbf{I}}{h} \rightarrow \mathbf{A} \text { weakly in } W^{1,2}\left((0, L) ; \mathbb{R}^{3 \times 3}\right) .
$$

In particular, $\mathbf{A} \in W^{1,2}\left((0, L) ; \mathbb{R}^{3 \times 3}\right)$ and $h^{-1}\left(\widetilde{\mathbf{R}}^{h}-\mathbf{I}\right)$ also converges uniformly. Using (4.22) we deduce that

$$
\mathbf{A}^{h} \rightarrow \mathbf{A} \text { uniformly. }
$$

In view of (4.12) this clearly implies the convergence property in (d). Since $\mathbf{R}^{h} \in$ $\mathrm{SO}(3)$ we have

$$
\mathbf{A}^{h}+\left(\mathbf{A}^{h}\right)^{T}=-h \mathbf{A}^{h}\left(\mathbf{A}^{h}\right)^{T}
$$

Hence, $\mathbf{A}+\mathbf{A}^{T}=0$. Moreover, after division by $2 h$ we obtain property (e) by (4.26). For adapting the proof to the proof of Theorem 2.2 in [25] it is essential to see

$$
\begin{aligned}
\left(\nabla \widetilde{\boldsymbol{y}}^{h}\right) \circ \boldsymbol{\Theta}^{h} \circ P^{h} & =\left(\nabla\left(\widetilde{\boldsymbol{y}}^{h} \circ \boldsymbol{\Theta}^{h}\right) \circ P^{h}\right)\left(\left(\nabla \boldsymbol{\Theta}^{h}\right)^{-1} \circ P^{h}\right) \\
& =\nabla_{h}\left(\widetilde{\boldsymbol{y}}^{h} \circ \boldsymbol{\Theta}^{h} \circ P^{h}\right)\left(\left(\nabla \boldsymbol{\Theta}^{h}\right)^{-1} \circ P^{h}\right) .
\end{aligned}
$$

From (4.27) it follows

$$
\left(\left(\nabla \widetilde{\boldsymbol{y}}^{h}\right) \circ \boldsymbol{\Theta}^{h} \circ P^{h}\right)\left(\left(\nabla \boldsymbol{\Theta}^{h}\right) \circ P^{h}\right)=\nabla_{h}\left(\widetilde{\boldsymbol{y}}^{h} \circ \boldsymbol{\Theta}^{h} \circ P^{h}\right) .
$$


and

$$
\begin{aligned}
\left(\nabla \widetilde{\boldsymbol{y}}^{h}\right) \circ \boldsymbol{\Theta}^{h} \circ P^{h}-\mathbf{I}= & \left(\nabla_{h}\left(\widetilde{\boldsymbol{y}}^{h} \circ \boldsymbol{\Theta}^{h} \circ P^{h}-\boldsymbol{\Theta}^{h} \circ P^{h}\right)\right)\left(\left(\nabla \boldsymbol{\Theta}^{h}\right)^{-1} \circ P^{h}-\mathbf{R}_{e}^{T}\right) \\
& +\left(\nabla_{h}\left(\widetilde{\boldsymbol{y}}^{h} \circ \boldsymbol{\Theta}^{h} \circ P^{h}-\boldsymbol{\Theta}^{h} \circ P^{h}\right)\right) \mathbf{R}_{e}^{T} .
\end{aligned}
$$

Let us notice that from (2.8), (3.5), (3.6) we can conclude

$$
\boldsymbol{\Theta}_{k}=h \theta_{k}+h x_{k}^{\prime}+O_{k}\left(h^{3}\right) \text { for } k=2,3,
$$

where $\left\|O_{k}\left(h^{3}\right)\right\|_{C^{1}(\Omega)} \leq C h^{3}$.

By multiplying (d) with $\left(\nabla \Theta^{h}\right) \circ P^{h}=\nabla_{h}\left(\Theta^{h} \circ P^{h}\right)$ and using (3.9), (4.28) we obtain

$$
\frac{\nabla_{h}\left(\widetilde{\boldsymbol{y}}^{h} \circ \boldsymbol{\Theta}^{h} \circ P^{h}-\boldsymbol{\Theta}^{h} \circ P^{h}\right)}{h} \rightarrow \mathbf{A} \mathbf{R}_{e} \text { in } L^{2}(\Omega) .
$$

Property (b) immediately from (4.31) by using (3.7), (4.23) and (4.30). Moreover, $v_{k}^{\prime}=\mathbf{A}_{k 1}$ for $k=2,3$ so that $v_{k} \in W^{2,2}(0, L)$ since $\mathbf{A} \in W^{1,2}(0, L)$. By using (e), (3.10), (4.12) and (4.31) from (4.29) we conclude that

$$
\left\|\frac{1}{h^{2}} \operatorname{sym}\left(\nabla_{h}\left(\widetilde{\boldsymbol{y}}^{h} \circ \boldsymbol{\Theta}^{h} \circ P^{h}-\boldsymbol{\Theta}^{h} \circ P^{h}\right) \mathbf{R}_{e}^{T}\right)\right\|_{L^{2}\left((0, L) ; \mathbb{R}^{3 \times 3}\right)} \leq C
$$

The weak convergence of $u^{h}$ follows from (3.7), (4.32) and the definition of $\mathbf{R}_{e}$. By using the convergence (4.31) and Poincare inequality on each cut $\left\{x_{1}\right\} \times \omega$ we can conclude

$$
\begin{gathered}
\frac{\widetilde{\boldsymbol{y}}_{2}^{h} \circ \boldsymbol{\Theta}^{h} \circ P^{h}-\boldsymbol{\Theta}_{2}^{h} \circ P^{h}}{h^{2}}-\frac{1}{h^{2} A} \int_{\omega}\left(\widetilde{\boldsymbol{y}}_{2}^{h} \circ \boldsymbol{\Theta}_{2}^{h} \circ P^{h}-\boldsymbol{\Theta}_{2}^{h} \circ P^{h}\right) \\
\rightarrow\left(\mathbf{A R}_{e}\right)_{22} x_{2}+\left(\mathbf{A R}_{e}\right)_{23} x_{3} \text { in } L^{2}(\Omega) .
\end{gathered}
$$

By using (2.3), (4.7) and (4.30) we conclude from (4.33)

$$
w_{2}^{h}:=\frac{\frac{1}{h} \widetilde{\boldsymbol{y}}_{2}^{h} \circ \boldsymbol{\Theta}^{h} \circ P^{h}-x_{2}^{\prime}}{h}-\frac{1}{h^{2} A} \int_{\omega} \widetilde{\boldsymbol{y}}_{2}^{h} \circ \boldsymbol{\Theta}^{h} \circ P^{h} \rightarrow \mathbf{A}_{23} x_{3}^{\prime} \text { in } L^{2}(\Omega) .
$$

Let us note that since the left hand side of (4.33) i.e. (4.34) is bounded in $W^{1,2}(\Omega)$ the convergence in (4.34) is in fact weak in $W^{1,2}(\Omega)$. The only nontrivial thing to prove is the boundedness of $\partial_{1} w_{2}^{h}$ in $L^{2}(\Omega)$. By the chain rule we have for $i=1,2,3$

$$
\begin{gathered}
\partial_{1}\left(\widetilde{\boldsymbol{y}}_{i}^{h} \circ \boldsymbol{\Theta}^{h} \circ P^{h}\right)=\left(\left(\partial_{1} \widetilde{\boldsymbol{y}}_{i}^{h}\right) \circ \boldsymbol{\Theta}^{h} \circ P^{h}\right)\left(\left(\partial_{1} \boldsymbol{\Theta}_{1}^{h}\right) \circ P^{h}\right) \\
+\left(\left(\partial_{2} \widetilde{\boldsymbol{y}}_{i}^{h}\right) \circ \boldsymbol{\Theta}^{h} \circ P^{h}\right)\left(\left(\partial_{1} \boldsymbol{\Theta}_{2}^{h}\right) \circ P^{h}\right)+\left(\left(\partial_{3} \widetilde{\boldsymbol{y}}_{i}^{h}\right) \circ \boldsymbol{\Theta}^{h} \circ P^{h}\right)\left(\left(\partial_{1} \boldsymbol{\Theta}_{3}^{h}\right) \circ P^{h}\right)
\end{gathered}
$$

and for $k=2,3$

$$
\begin{gathered}
\partial_{k}\left(\widetilde{\boldsymbol{y}}_{i}^{h} \circ \boldsymbol{\Theta}^{h} \circ P^{h}\right)=h\left[\left(\left(\partial_{1} \widetilde{\boldsymbol{y}}_{i}^{h}\right) \circ \boldsymbol{\Theta}^{h} \circ P^{h}\right)\left(\left(\partial_{k} \boldsymbol{\Theta}_{1}^{h}\right) \circ P^{h}\right)\right. \\
\left.+\left(\left(\partial_{2} \widetilde{\boldsymbol{y}}_{i}^{h}\right) \circ \boldsymbol{\Theta}^{h} \circ P^{h}\right)\left(\left(\partial_{k} \boldsymbol{\Theta}_{2}^{h}\right) \circ P^{h}\right)+\left(\left(\partial_{3} \widetilde{\boldsymbol{y}}_{i}^{h}\right) \circ \boldsymbol{\Theta}^{h} \circ P^{h}\right)\left(\left(\partial_{k} \boldsymbol{\Theta}_{3}^{h}\right) \circ P^{h}\right)\right]
\end{gathered}
$$


From $(4.12),(4.34)$ and $(4.35)$ we conclude that the boundedness of $\partial_{1} w_{2}^{h}$ in $L^{2}(\Omega)$ is equivalent to the boundedness of

$$
\begin{aligned}
z_{2}^{h}= & \frac{\mathbf{R}_{21}^{h} \partial_{1} \boldsymbol{\Theta}_{1}+\mathbf{R}_{22}^{h} \partial_{1} \boldsymbol{\Theta}_{2}+\mathbf{R}_{23}^{h} \partial_{1} \boldsymbol{\Theta}_{3}-h\left(p_{2}^{\prime} x_{2}-p_{3}^{\prime} x_{3}\right)}{h^{2}} \\
& -\frac{1}{h^{2} A}\left(\mathbf{R}_{21}^{h} \int_{\omega} \partial_{1} \boldsymbol{\Theta}_{1}+\mathbf{R}_{22}^{h} \int_{\omega} \partial_{1} \boldsymbol{\Theta}_{2}+\mathbf{R}_{23}^{h} \int_{\omega} \partial_{1} \boldsymbol{\Theta}_{3}\right),
\end{aligned}
$$

in $L^{2}(\Omega)$. By using (2.3) and (3.18) we conclude

$$
\begin{aligned}
z_{2}^{h}= & \frac{\mathbf{R}_{21}^{h}\left(x_{2}\left(\boldsymbol{n}_{1}^{h}\right)^{\prime}+x_{3}\left(\boldsymbol{b}_{1}^{h}\right)^{\prime}\right)+\mathbf{R}_{22}^{h}\left(x_{2}\left(\boldsymbol{n}_{2}^{h}\right)^{\prime}+x_{3}\left(\boldsymbol{b}_{2}^{h}\right)^{\prime}\right)}{h} \\
& +\frac{\mathbf{R}_{23}^{h}\left(x_{2}\left(\boldsymbol{n}_{3}^{h}\right)^{\prime}+x_{3}\left(\boldsymbol{b}_{3}^{h}\right)^{\prime}\right)-\left(p_{2}^{\prime} x_{2}-p_{3}^{\prime} x_{3}\right)}{h} .
\end{aligned}
$$

The boundedness of $z_{2}^{h}$ in $L^{2}(\Omega)$ is the consequence of (3.5), (3.6) and (4.14). Now we have proved $w_{2}^{h} \rightarrow \mathbf{A}_{23} x_{3}^{\prime}$ weakly in $W^{1,2}(\Omega)$.

Analogously we conclude

$$
w_{3}^{h}:=\frac{\frac{1}{h} \widetilde{\boldsymbol{y}}_{3}^{h} \circ \boldsymbol{\Theta}^{h} \circ P^{h}-x_{3}^{\prime}}{h}-\frac{1}{h^{2} A} \int_{\omega} \widetilde{\boldsymbol{y}}_{3}^{h} \circ \boldsymbol{\Theta}^{h} \circ P^{h} \rightarrow-\mathbf{A}_{23} x_{2}^{\prime}, .
$$

weakly in $W^{1,2}(\Omega)$. Now, since $w^{h}$ can be written as

$$
w^{h}\left(x_{1}\right)=\frac{1}{A \mu(\omega)} \int_{\omega}\left(x_{2}^{\prime} w_{3}^{h}-x_{3}^{\prime} w_{2}^{h}\right) d x_{2} d x_{3}
$$

it is clear that $w^{h}$ converges weakly to the function $w=-\mathbf{A}_{23}=\mathbf{A}_{32}$ in $W^{1,2}(0, L)$. Let us define for $\boldsymbol{\beta}^{h}: \Omega^{\prime} \rightarrow \mathbb{R}^{3}, \boldsymbol{\beta}^{h}=\boldsymbol{\gamma} \circ \boldsymbol{x}^{\prime-1}$. By the chain rule we have

$$
\begin{aligned}
\partial_{1} \boldsymbol{\beta}_{i}^{h}= & \left(\partial_{1} \boldsymbol{\gamma}_{i}^{h}\right) \circ\left(\boldsymbol{x}^{\prime}\right)^{-1}+\left(p_{2}^{\prime} x_{2}^{\prime}+p_{3}^{\prime} x_{3}^{\prime}\right)\left(\partial_{2} \boldsymbol{\gamma}_{i}^{h}\right) \circ\left(\boldsymbol{x}^{\prime}\right)^{-1} \\
& +\left(-p_{3}^{\prime} x_{2}^{\prime}+p_{2}^{\prime} x_{3}^{\prime}\right)\left(\partial_{3} \boldsymbol{\gamma}_{i}^{h}\right) \circ\left(\boldsymbol{x}^{\prime}\right)^{-1} \\
\partial_{2} \boldsymbol{\beta}_{i}^{h}= & p_{2}\left(\partial_{2} \boldsymbol{\gamma}_{i}^{h}\right) \circ\left(\boldsymbol{x}^{\prime}\right)^{-1}-p_{3}\left(\partial_{3} \boldsymbol{\gamma}_{i}^{h}\right) \circ\left(\boldsymbol{x}^{\prime}\right)^{-1} \\
\partial_{3} \boldsymbol{\beta}_{i}^{h}= & p_{3}\left(\partial_{2} \boldsymbol{\gamma}_{i}^{h}\right) \circ\left(\boldsymbol{x}^{\prime}\right)^{-1}+p_{2}\left(\partial_{3} \boldsymbol{\gamma}_{i}^{h}\right) \circ\left(\boldsymbol{x}^{\prime}\right)^{-1} .
\end{aligned}
$$

By differentiating $\boldsymbol{\beta}_{1}$ with respect to $x_{k}^{\prime}$, with $\mathrm{k}=2,3$, we have

$$
\begin{aligned}
& \partial_{2} \boldsymbol{\beta}_{1}^{h}=\frac{1}{h^{3}} \partial_{2}\left(\widetilde{\boldsymbol{y}}_{1}^{h} \circ \boldsymbol{\Theta}^{h} \circ P^{h} \circ\left(\boldsymbol{x}^{\prime}\right)^{-1}\right)+\frac{1}{h}\left(\left(v_{2}^{h}\right)^{\prime}+\theta_{2}^{\prime}\right), \\
& \left.\partial_{3} \boldsymbol{\beta}_{1}^{h}=\frac{1}{h^{3}} \partial_{3}\left(\widetilde{\boldsymbol{y}}_{1}^{h} \circ \boldsymbol{\Theta}^{h} \circ P^{h} \circ\left(\boldsymbol{x}^{\prime}\right)^{-1}\right)+\frac{1}{h}\left(\left(v_{3}^{h}\right)^{\prime}+\theta_{3}^{\prime}\right)\right) .
\end{aligned}
$$

Let us analyze only $\partial_{2} \boldsymbol{\beta}_{1}^{h}$. We have by $(3.18),(4.36)$ and the chain rule

$$
\begin{aligned}
\partial_{2} \boldsymbol{\beta}_{1}^{h}= & \frac{\left(\left(\partial_{1} \widetilde{\boldsymbol{y}}_{1}^{h}\right) \circ \boldsymbol{\Theta}^{h} \circ P^{h} \circ\left(\boldsymbol{x}^{\prime}\right)^{-1}\right)\left(p_{2} \boldsymbol{n}_{1}^{h}-p_{3} \boldsymbol{b}_{1}^{h}\right)}{h^{2}} \\
& +\frac{\left(\left(\partial_{2} \widetilde{\boldsymbol{y}}_{1}^{h}\right) \circ \boldsymbol{\Theta}^{h} \circ P^{h} \circ\left(\boldsymbol{x}^{\prime}\right)^{-1}\right)\left(p_{2} \boldsymbol{n}_{2}^{h}-p_{3} \boldsymbol{b}_{2}^{h}\right)}{h^{2}} \\
& +\frac{\left(\left(\partial_{3} \widetilde{\boldsymbol{y}}_{1}^{h}\right) \circ \boldsymbol{\Theta}^{h} \circ P^{h} \circ\left(\boldsymbol{x}^{\prime}\right)^{-1}\right)\left(p_{2} \boldsymbol{n}_{3}^{h}-p_{3} \boldsymbol{b}_{3}^{h}\right)}{h^{2}} \\
& +\frac{1}{h}\left(\left(v_{2}^{h}\right)^{\prime}+\theta_{2}^{\prime}\right) .
\end{aligned}
$$


By using (3.5), (3.6), (3.18), (4.12), (4.14), (4.35) and the definition of $v_{k}^{h}$ we can conclude that for proving the boundedness of $\partial_{2} \boldsymbol{\beta}_{1}^{h}$ it is enough to prove the boundedness of $\delta_{1,2}^{h}$ in $L^{2}(\Omega)$ where

$$
\delta_{1,2}^{h}:=\frac{-h \mathbf{R}_{11}^{h} \theta_{2}^{\prime}+\mathbf{R}_{12}^{h}}{h^{2}}+\frac{\mathbf{R}_{21}^{h}+h \theta_{2}^{\prime}}{h^{2}}=\frac{1-\mathbf{R}_{11}^{h}}{h} \theta_{2}^{\prime}+\frac{\mathbf{R}_{12}^{h}+\mathbf{R}_{21}^{h}}{h^{2}} .
$$

The boundedness of $\delta_{1,2}^{h}$ in $L^{\infty}(\Omega)$ is then the consequence of the property (e). In the same way we can prove the boundedness of $\partial_{3} \boldsymbol{\beta}_{1}^{h}$. Using the Poincare inequality and the fact that $\int_{\omega^{\prime}\left(x_{1}\right)} \boldsymbol{\beta}_{1}^{h} d x_{2}, d x_{3}^{\prime}=0$, we deduce that there exists a constant $C>0$ such that

$$
\int_{\omega^{\prime}\left(x_{1}\right)}\left(\boldsymbol{\beta}_{1}^{h}(x)\right)^{2} d x_{2} d x_{3} \leq C \int_{\omega^{\prime}\left(x_{1}\right)}\left[\left(\partial_{2} \boldsymbol{\beta}_{1}^{h}(x)\right)^{2}+\left(\partial_{3} \boldsymbol{\beta}_{1}^{h}(x)\right)^{2}\right] d x_{2} d x_{3}
$$

for a.e. $x_{1} \in(0, L)$ and for every $h$. Although the constant $C$ depends on the domain, since all domains are translations and rotations of the domain $\omega$, the constant $C$ can be chosen uniformly. Integrating both sides with respect to $x_{1}$, we obtain that the sequence $\left(\boldsymbol{\beta}_{1}^{h}\right)$ is bounded in $L^{2}\left(\Omega^{\prime}\right)$ so, up to subsequences $\boldsymbol{\beta}_{1}^{h} \rightarrow \boldsymbol{\beta}_{1}$ and $\partial_{k} \boldsymbol{\beta}_{1}^{h} \rightarrow \partial_{k} \boldsymbol{\beta}_{1}$ weakly in $L^{2}\left(\Omega^{\prime}\right)$, for $k=2,3$. From the relations (4.41) it can be concluded that $\gamma_{1}^{h} \rightarrow \gamma_{1}$ and $\partial_{k} \gamma_{1}^{h} \rightarrow \partial_{k} \gamma_{1}$ weakly in $L^{2}(\Omega)$, for $k=2,3$, where $\boldsymbol{\gamma}=\boldsymbol{\beta} \circ \boldsymbol{x}^{\prime}$. For the sequences $\left(\boldsymbol{\beta}_{2}^{h}\right),\left(\boldsymbol{\beta}_{3}^{h}\right)$, we have by differentiation that for $j, k=2,3$

$$
\partial_{j} \boldsymbol{\beta}_{k}^{h}=\frac{1}{h^{2}}\left(\frac{1}{h} \partial_{j}\left(\widetilde{\boldsymbol{y}}_{k}^{h} \circ \boldsymbol{\Theta}^{h} \circ P^{h} \circ\left(\boldsymbol{x}^{\prime}\right)^{-1}\right)-h \delta_{j k}-h w^{h}\left(1-\delta_{j k}\right)(-1)^{k}\right) .
$$

By using the chain rule we see that for $k=2,3$,

$$
\begin{aligned}
\partial_{2}\left(\widetilde{\boldsymbol{y}}_{k}^{h} \circ \boldsymbol{\Theta}^{h} \circ P^{h} \circ\left(\boldsymbol{x}^{\prime}\right)^{-1}\right) & =h\left(\left(\left(\partial_{1} \widetilde{\boldsymbol{y}}_{k}^{h}\right) \circ \boldsymbol{\Theta}^{h} \circ P^{h} \circ\left(\boldsymbol{x}^{\prime}\right)^{-1}\right)\left(p_{2} \boldsymbol{n}_{1}^{h}-p_{3} \boldsymbol{b}_{1}^{h}\right)\right. \\
& +\left(\left(\partial_{2} \widetilde{\boldsymbol{y}}_{k}^{h}\right) \circ \boldsymbol{\Theta}^{h} \circ P^{h} \circ\left(\boldsymbol{x}^{\prime}\right)^{-1}\right)\left(p_{2} \boldsymbol{n}_{2}^{h}-p_{3} \boldsymbol{b}_{2}^{h}\right) \\
& \left.+\left(\left(\partial_{3} \widetilde{\boldsymbol{y}}_{k}^{h}\right) \circ \boldsymbol{\Theta}^{h} \circ P^{h} \circ\left(\boldsymbol{x}^{\prime}\right)^{-1}\right)\left(p_{2} \boldsymbol{n}_{3}^{h}-p_{3} \boldsymbol{b}_{3}^{h}\right)\right), \\
\partial_{3}\left(\widetilde{\boldsymbol{y}}_{k}^{h} \circ \boldsymbol{\Theta}^{h} \circ P^{h} \circ\left(\boldsymbol{x}^{\prime}\right)^{-1}\right) & =h\left(\left(\left(\partial_{1} \widetilde{\boldsymbol{y}}_{k}^{h}\right) \circ \boldsymbol{\Theta}^{h} \circ P^{h} \circ\left(\boldsymbol{x}^{\prime}\right)^{-1}\right)\left(p_{3} \boldsymbol{n}_{1}^{h}+p_{2} \boldsymbol{b}_{1}^{h}\right)\right. \\
& +\left(\left(\partial_{2} \widetilde{\boldsymbol{y}}_{k}^{h}\right) \circ \boldsymbol{\Theta}^{h} \circ P^{h} \circ\left(\boldsymbol{x}^{\prime}\right)^{-1}\right)\left(p_{3} \boldsymbol{n}_{2}^{h}+p_{2} \boldsymbol{b}_{2}^{h}\right) \\
& \left.+\left(\left(\partial_{3} \widetilde{\boldsymbol{y}}_{k}^{h}\right) \circ \boldsymbol{\Theta}^{h} \circ P^{h} \circ\left(\boldsymbol{x}^{\prime}\right)^{-1}\right)\left(p_{3} \boldsymbol{n}_{3}^{h}+p_{2} \boldsymbol{b}_{3}^{h}\right)\right) .
\end{aligned}
$$

Now we want to check that for $j, k=2,3$

$$
e_{j k}\left(\boldsymbol{\beta}^{h}\right):=\frac{1}{2}\left(\partial_{j} \boldsymbol{\beta}_{k}^{h}+\partial_{k} \boldsymbol{\beta}_{j}^{h}\right)
$$

is bounded in $L^{2}\left(\Omega^{\prime}\right)$. In the similar way as for $\boldsymbol{\beta}_{1}$ (relations (4.44) and (4.45)) we can using (3.5), (3.6), (4.12), (4.14) and the property (e) conclude that for every 
$j, k=2,3, e_{j k}\left(\boldsymbol{\beta}^{h}\right) \in L^{2}\left(\Omega^{\prime}\right)$. By using Korn's inequality (Lemma 1) we have that there exists $C>0$ such that

$$
\begin{aligned}
& \left\|\boldsymbol{\beta}_{2}^{h}\right\|_{W^{1,2}\left(\omega^{\prime}\left(x_{1}\right)\right)}^{2}+\left\|\boldsymbol{\beta}_{3}^{h}\right\|_{W^{1,2}\left(\omega^{\prime}\left(x_{1}\right)\right)}^{2} \leq C\left(\left|\int_{\omega^{\prime}\left(x_{1}\right)} \boldsymbol{\beta}_{2}^{h} d x_{2}^{\prime} d x_{3}^{\prime}\right|\right. \\
& \quad+\left|\int_{\omega^{\prime}\left(x_{1}\right)} \boldsymbol{\beta}_{3}^{h} d x_{2}^{\prime} d x_{3}^{\prime}\right|+\left|\int_{\omega^{\prime}\left(x_{1}\right)}\left(x_{3}^{\prime} \boldsymbol{\beta}_{2}^{h}-x_{2}^{\prime} \boldsymbol{\beta}_{3}^{h}\right) d x_{2}^{\prime} d x_{3}^{\prime}\right| \\
& \left.\quad+\sum_{j, k=1,2}\left\|e_{j k}\left(\boldsymbol{\beta}^{h}\right)\right\|_{L^{2}\left(\omega^{\prime}\left(x_{1}\right)\right)}\right),
\end{aligned}
$$

for a.e. $x_{1} \in(0, L)$. From the definition of $v_{k}^{h}$ and $w^{h}$ we see that the functions $\left(\boldsymbol{\beta}_{2}^{h}\left(x_{1}, \cdot\right), \boldsymbol{\beta}_{3}^{h}\left(x_{1}, \cdot\right)\right)$ belong to the space

$$
\begin{gathered}
\mathcal{B}_{x_{1}}=\left\{\boldsymbol{\beta}=\left(\boldsymbol{\beta}_{2}, \boldsymbol{\beta}_{3}\right) \in W^{1,2}\left(\omega^{\prime}\left(x_{1}\right) ; \mathbb{R}^{2}\right): \int_{\omega^{\prime}\left(x_{1}\right)} \boldsymbol{\beta} d x_{2}^{\prime} d x_{3}^{\prime}=0,\right. \\
\left.\int_{\omega^{\prime}\left(x_{1}\right)}\left(x_{2}^{\prime} \boldsymbol{\beta}_{3}-x_{3}^{\prime} \boldsymbol{\beta}_{2}\right) d x_{2}^{\prime} d x_{3}^{\prime}=0\right\}
\end{gathered}
$$

for every $x_{1}$. By integrating (4.48) with respect to $x_{1}$ we conclude that $\boldsymbol{\beta}_{2}^{h}, \boldsymbol{\beta}_{3}^{h}$ are bounded in $L^{2}\left(\Omega^{\prime}\right)$ as well as their derivatives with respect to $x_{2}, x_{3}$. From this we can conclude the same fact about $\gamma_{2}^{h}, \gamma_{3}^{h}$. The fact that the weak limit belongs to the space $\mathcal{C}$ can be concluded from the fact that for every $h$ and a.e. $x_{1}$ $\left(\boldsymbol{\beta}_{2}^{h}\left(x_{1}, \cdot\right), \boldsymbol{\beta}_{3}^{h}\left(x_{1}, \cdot\right)\right) \in \mathcal{B}_{x_{1}}$. This finishes the proof of (f).

\subsection{Lower bound}

Lemma 2 Let $\boldsymbol{y}^{h}, \widetilde{\boldsymbol{y}}^{h}, E^{h}, \mathbf{R}^{h}, u^{h}, v^{h}, w^{h}, \boldsymbol{\gamma}^{h}, \boldsymbol{\beta}^{h}=\boldsymbol{\gamma}^{h} \circ\left(\boldsymbol{x}^{\prime}\right)^{-1}, \boldsymbol{\gamma}, \boldsymbol{\beta}=\boldsymbol{\gamma} \circ\left(\boldsymbol{x}^{\prime}\right)^{-1}$, $\mathbf{A}$ be as in Theorem 3 and let us suppose that the condition (4.11) is satisfied and that $\boldsymbol{\gamma}^{h} \rightarrow \boldsymbol{\gamma}, \partial_{2} \boldsymbol{\gamma}^{h} \rightarrow \partial_{2} \boldsymbol{\gamma}, \partial_{3} \boldsymbol{\gamma}^{h} \rightarrow \partial_{3} \boldsymbol{\gamma}$ weakly in $L^{2}(\Omega)$ i.e. $\boldsymbol{\beta}^{h} \rightarrow \boldsymbol{\beta}, \partial_{2} \boldsymbol{\beta}^{h} \rightarrow \partial_{2} \boldsymbol{\beta}$, $\partial_{3} \boldsymbol{\beta}^{h} \rightarrow \partial_{3} \boldsymbol{\beta}$ weakly in $L^{2}\left(\Omega^{\prime}\right)$. Let us define

$$
\begin{aligned}
\boldsymbol{\eta}_{1}^{h}(x)=\frac{1}{h}( & \frac{\left(\widetilde{\boldsymbol{y}}_{1}^{h} \circ \boldsymbol{\Theta}^{h} \circ P^{h}\right)(x)-\boldsymbol{\Theta}_{1}^{h} \circ P^{h}}{h^{2}}-u^{h}\left(x_{1}\right) \\
& \left.+x_{2}^{\prime}\left(v_{2}^{h}\right)^{\prime}\left(x_{1}\right)+x_{3}^{\prime}\left(v_{3}^{h}\right)^{\prime}\left(x_{1}\right)\right), \\
\boldsymbol{\eta}_{k}^{h}(x)=\frac{1}{h^{2}}( & \frac{\left(\widetilde{\boldsymbol{y}}_{k}^{h} \circ \boldsymbol{\Theta}^{h} \circ P^{h}\right)(x)-\boldsymbol{\Theta}_{k}^{h} \circ P^{h}}{h} \\
& \left.-v_{k}^{h}\left(x_{1}\right)-h\left(x_{k}^{\prime}\right)^{\perp} \omega^{h}\left(x_{1}\right)\right), \text { for } k=2,3,
\end{aligned}
$$

and $\boldsymbol{\kappa}^{h}=\boldsymbol{\eta}^{h} \circ\left(\boldsymbol{x}^{\prime}\right)^{-1}$. Then we have that $\boldsymbol{\eta}^{h} \rightarrow \boldsymbol{\eta}$ weakly in $L^{2}(\Omega)$ and $\partial_{k} \boldsymbol{\eta}^{h} \rightarrow \partial_{k} \boldsymbol{\eta}$ weakly in $L^{2}(\Omega)$ i.e. $\boldsymbol{\kappa}^{h} \rightarrow \boldsymbol{\kappa}, \partial_{2} \boldsymbol{\kappa}^{h} \rightarrow \partial_{2} \boldsymbol{\kappa}, \partial_{3} \boldsymbol{\kappa}^{h} \rightarrow \partial_{3} \boldsymbol{\kappa}$ weakly in $L^{2}\left(\Omega^{\prime}\right)$. Here

$$
\begin{aligned}
\boldsymbol{\eta}_{1} & =\gamma_{1}, \\
\boldsymbol{\eta}_{2} & =\gamma_{2}+f_{2}^{2} x_{2}+f_{2}^{3} x_{3}=\gamma_{2}+g_{2}^{2} x_{2}^{\prime}+g_{2}^{3} x_{3}^{\prime}, \\
\boldsymbol{\eta}_{3} & =\gamma_{3}+f_{3}^{2} x_{2}+f_{3}^{3} x_{3}=\gamma_{3}+g_{3}^{2} x_{2}^{\prime}+g_{3}^{3} x_{3}^{\prime}, \\
\boldsymbol{\kappa} & =\boldsymbol{\eta} \circ\left(\boldsymbol{x}^{\prime}\right)^{-1},
\end{aligned}
$$


$f_{k}^{j}$ are defined in Remark 2 and $g_{k}^{j}$ can be easily defined for the above identities to be valid i.e. for $k=2,3$, we define

$$
g_{k}^{2}=p_{2} f_{k}^{2}-p_{3} f_{k}^{3}, g_{k}^{3}=p_{3} f_{k}^{2}+p_{2} f_{k}^{3} .
$$

The following strain convergence is valid

$$
\mathbf{G}^{h}:=\frac{\left(\mathbf{R}^{h}\right)^{T}\left(\left(\nabla \boldsymbol{y}^{h}\right) \circ \boldsymbol{\Theta}^{h} \circ P^{h}\right)-\mathbf{I}}{h^{2}} \rightarrow \mathbf{G} \quad \text { in } L^{2}\left(\Omega ; \mathbb{R}^{3 \times 3}\right) .
$$

and the symmetric part of $\mathbf{G}$ denoted by $\widetilde{\mathbf{G}}$, satisfies

$$
\widetilde{\mathbf{G}}=\operatorname{sym}\left(\mathbf{J}-\frac{1}{2} \mathbf{A}^{2}+\mathbf{K}\right)
$$

where

$$
\begin{array}{r}
\mathbf{J}=\left(\begin{array}{ccc}
u^{\prime}+v_{2}^{\prime} \theta_{2}^{\prime}+v_{3}^{\prime} \theta_{3}^{\prime} & 0 & 0 \\
w \theta_{3}^{\prime} & v_{2}^{\prime} \theta_{2}^{\prime} & v_{2}^{\prime} \theta_{3}^{\prime} \\
-w \theta_{2}^{\prime} & v_{3}^{\prime} \theta_{2}^{\prime} & v_{3}^{\prime} \theta_{3}^{\prime}
\end{array}\right) \\
\mathbf{K}=\left(\begin{array}{c|c|c}
-x_{2}^{\prime} v_{2}^{\prime \prime}-x_{3}^{\prime} v_{3}^{\prime \prime} & & \\
-x_{3}^{\prime} w^{\prime} & \partial_{2} \kappa & \partial_{3} \kappa \\
x_{2}^{\prime} w^{\prime} & &
\end{array}\right) .
\end{array}
$$

Moreover,

$$
\begin{aligned}
\liminf _{h \rightarrow 0} \frac{1}{h^{6}} \int_{\hat{\Omega}^{h}} W^{h}\left(x, \nabla \hat{\boldsymbol{y}}^{h}\right) d x & =\liminf _{h \rightarrow 0} \frac{1}{h^{4}} \int_{\Omega} W\left(x^{h},\left(\nabla \hat{\boldsymbol{y}}^{h}\right) \circ \boldsymbol{\Theta}^{h} \circ P^{h}\right) d x^{h} \\
& \geq \frac{1}{2} \int_{\Omega} Q_{3}(x, \widetilde{\mathbf{G}}(x)) d x
\end{aligned}
$$

where $Q_{3}$ is twice the quadratic form of linearized elasticity, i.e.,

$$
Q_{3}(x, \mathbf{F})=\frac{\partial^{2} W}{\partial \mathbf{F}^{2}}(\mathbf{I})[\mathbf{F}, \mathbf{F}]
$$

Proof. We follow the proof of Lemma 2.3 in [25]. Firstly, using Remark 1, it can be seen that

$$
\begin{aligned}
\boldsymbol{\eta}_{1}^{h} & =\gamma_{1}^{h}+h \boldsymbol{o}_{1}, \\
\boldsymbol{\eta}_{2}^{h} & =\gamma_{2}^{h}+f_{2}^{2} x_{2}+f_{2}^{3} x_{3}+h \boldsymbol{o}_{2}, \\
\boldsymbol{\eta}_{3}^{h} & =\gamma_{3}^{h}+f_{3}^{2} x_{2}+f_{3}^{3} x_{3}+h \boldsymbol{o}_{3},
\end{aligned}
$$

where $\left\|\boldsymbol{o}_{i}\right\|_{C^{1}(\Omega)} \leq C$, for some $C>0$. The convergence of $\boldsymbol{\eta}^{h}$ is an easy consequence of the convergence of $\gamma^{h}$. The estimate (4.12) implies that the $L^{2}$ norm of $\mathbf{G}^{h}$ is bounded; therefore up to subsequences, there exists $\mathbf{G} \in L^{2}\left(\Omega ; \mathbb{R}^{3 \times 3}\right)$ such that (4.57) is satisfied. In order to identify the symmetric part of $\mathbf{G}$ we decompose $\mathbf{R}^{h} \mathbf{G}^{h}$ as follows:

$$
\mathbf{R}^{h} \mathbf{G}^{h}=\frac{\left(\nabla \widetilde{\boldsymbol{y}}^{h}\right) \circ \boldsymbol{\Theta}^{h} \circ P^{h}-\mathbf{I}}{h^{2}}-\frac{\mathbf{R}^{h}-\mathbf{I}}{h^{2}},
$$

so that

$$
\mathbf{F}^{h}:=\operatorname{sym} \frac{\left(\nabla \widetilde{\boldsymbol{y}}^{h}\right) \circ \boldsymbol{\Theta}^{h} \circ P^{h}-\mathbf{I}}{h^{2}}=\operatorname{sym}\left(\mathbf{R}^{h} \mathbf{G}^{h}\right)+\operatorname{sym} \frac{\mathbf{R}^{h}-\mathbf{I}}{h^{2}} .
$$


The right hand side converges weakly to $\widetilde{\mathbf{G}}+\frac{\mathbf{A}^{2}}{2}$ by $(4.14),(4.57)$ and property (e) of the Theorem 3. Therefore the sequence $\mathbf{F}^{h}$ has a weak limit in $L^{2}(0, L)$, satisfying $\mathbf{F}=\widetilde{\mathbf{G}}+\frac{\mathbf{A}^{2}}{2}$. To conclude we only need to identify $\mathbf{F}$. Consider the functions

$$
\phi_{1}^{h}:=\frac{\widetilde{\boldsymbol{y}}_{1}^{h} \circ \boldsymbol{\Theta}^{h} \circ P^{h}-x_{1}}{h^{2}} .
$$

From property (f) of Theorem 3 it follows that the functions $\phi_{1}^{h}-u^{h}+x_{2}^{\prime}\left(\left(v_{2}^{h}\right)^{\prime}+\right.$ $\left.\theta_{2}^{\prime}\right)+x_{3}^{\prime}\left(\left(v_{3}^{h}\right)^{\prime}+\theta_{3}^{\prime}\right)$, which are equal to $h \gamma_{1}^{h}$ converge strongly to 0 in $L^{2}(\Omega)$. Thus by property (a) and (b) of Theorem 3 we conclude that

$$
\phi_{1}^{h} \rightarrow u-x_{2}^{\prime}\left(v_{2}^{\prime}+\theta_{2}^{\prime}\right)-x_{3}^{\prime}\left(v_{3}^{\prime}+\theta_{3}^{\prime}\right) \text { in } L^{2}(\Omega) .
$$

By using the chain rule, the property (d) of Theorem 3, (3.5), (3.6), (4.30) we can conclude that

$\partial_{1} \phi_{1}^{h} \rightarrow \mathbf{F}_{11}-x_{2}\left(\theta_{2}^{\prime} p_{2}+\theta_{3}^{\prime} p_{3}\right)^{\prime}+x_{3}\left(\theta_{2}^{\prime} p_{3}-\theta_{3}^{\prime} p_{2}\right)^{\prime}-v_{2}^{\prime}\left(\partial_{1} x_{2}^{\prime}+\theta_{2}^{\prime}\right)-v_{3}^{\prime}\left(\partial_{1} x_{3}^{\prime}+\theta_{3}^{\prime}\right)$,

weakly in $L^{2}(\Omega)$. From (4.65) and (4.66) we conclude that

$$
\begin{aligned}
& u^{\prime}-\partial_{1} x_{2}^{\prime}\left(v_{2}^{\prime}+\theta_{2}^{\prime}\right)-x_{2}^{\prime}\left(v_{2}^{\prime \prime}+\theta_{2}^{\prime \prime}\right)-\partial_{1} x_{3}^{\prime}\left(v_{3}^{\prime}+\theta_{3}^{\prime}\right)-x_{3}^{\prime}\left(v_{3}^{\prime \prime}+\theta_{3}^{\prime \prime}\right) \\
= & \mathbf{F}_{11}-x_{2}\left(\theta_{2}^{\prime} p_{2}+\theta_{3}^{\prime} p_{3}\right)^{\prime}+x_{3}\left(\theta_{2}^{\prime} p_{3}-\theta_{3}^{\prime} p_{2}\right)^{\prime} \\
& -v_{2}^{\prime}\left(\partial_{1} x_{2}^{\prime}+\theta_{2}^{\prime}\right)-v_{3}^{\prime}\left(\partial_{1} x_{3}^{\prime}+\theta_{3}^{\prime}\right) .
\end{aligned}
$$

After some calculation we obtain

$$
\mathbf{F}_{11}=u^{\prime}+v_{2}^{\prime} \theta_{2}^{\prime}+v_{3}^{\prime} \theta_{3}^{\prime}-x_{2}^{\prime} v_{2}^{\prime \prime}-x_{3}^{\prime} v_{3}^{\prime \prime}
$$

To identify $\mathbf{F}_{12}$ we have to do some straight forward computations. By using the chain rule, (3.5), (3.6), Remark 1, property (d) of Theorem (3) we can conclude

$$
\begin{aligned}
& \frac{1}{h^{2}} \partial_{1}\left(\widetilde{\boldsymbol{y}}_{2}^{h} \circ \boldsymbol{\Theta}^{h} \circ P^{h}\right)+\frac{1}{h^{3}}\left(p_{2} \partial_{2}\left(\widetilde{\boldsymbol{y}}_{1}^{h} \circ \boldsymbol{\Theta}^{h} \circ P^{h}\right)-p_{3} \partial_{3}\left(\widetilde{\boldsymbol{y}}_{1}^{h} \circ \boldsymbol{\Theta}^{h} \circ P^{h}\right)\right)= \\
& 2 \mathbf{F}_{12}^{h}+\frac{\partial_{1} x_{2}^{\prime}}{h}-w\left(\theta_{3}^{\prime}+\partial_{1} x_{3}^{\prime}\right)+\mathbf{O}_{1}^{h},
\end{aligned}
$$

where $\lim _{h \rightarrow 0}\left\|\mathbf{O}_{1}^{h}\right\|_{L^{2}\left(\Omega ; \mathbb{R}^{3 \times 3}\right)}=0$. On the other hand it can be easily seen that

$$
\begin{aligned}
\partial_{2} \boldsymbol{\beta}_{1}^{h}= & p_{2} \partial_{2} \gamma_{1}^{h}-p_{3} \partial_{3} \gamma_{1}^{h} \\
= & \frac{1}{h^{3}}\left(p_{2} \partial_{2}\left(\widetilde{\boldsymbol{y}}_{1}^{h} \circ \boldsymbol{\Theta}^{h} \circ P^{h}\right)-p_{3} \partial_{3}\left(\widetilde{\boldsymbol{y}}_{1}^{h} \circ \boldsymbol{\Theta}^{h} \circ P^{h}\right)\right) \\
& +\frac{1}{h}\left(\left(v_{2}^{h}\right)^{\prime}+\theta_{2}^{\prime}\right) .
\end{aligned}
$$

From (4.34), (4.69), (4.70) we conclude

$$
\begin{aligned}
2 \mathbf{F}_{12}^{h}= & \frac{1}{h^{2}} \partial_{1}\left(\widetilde{\boldsymbol{y}}_{2}^{h} \circ \boldsymbol{\Theta}^{h} \circ P^{h}-h x_{2}^{\prime}\right)-\frac{1}{h}\left(\left(v_{2}^{h}\right)^{\prime}+\theta_{2}^{\prime}\right) \\
& +\partial_{2} \boldsymbol{\beta}_{1}^{h}+w \theta_{3}^{\prime}+w \partial_{1} x_{3}^{\prime}-\mathbf{O}_{1}^{h} \\
= & \partial_{1} w_{2}^{h}+\partial_{2} \boldsymbol{\beta}_{1}^{h}+w \theta_{3}^{\prime}+w \partial_{1} x_{3}^{\prime}-\mathbf{O}_{1}^{h} .
\end{aligned}
$$


By using (4.34) we conclude that the right hand side of (4.71) converges in $W^{-1,2}(\Omega)$ to

$$
\partial_{1}\left(-w x_{3}^{\prime}\right)+\partial_{2} \boldsymbol{\beta}_{1}+w \theta_{3}^{\prime}+w \partial_{1} x_{3}^{\prime}=-x_{3}^{\prime} w^{\prime}+w \theta_{3}^{\prime}+\partial_{2} \boldsymbol{\kappa}_{1},
$$

since $\boldsymbol{\beta}_{1}=\boldsymbol{\kappa}_{1}$. On the other hand we know that the left hand side of (4.71) converges strongly in $L^{2}(\Omega)$ to $2 \mathbf{F}_{12}$ and thus we can conclude

$$
\mathbf{F}_{12}=\frac{1}{2}\left(-x_{3}^{\prime} w^{\prime}+w \theta_{3}^{\prime}+\partial_{2} \boldsymbol{\kappa}_{1}\right) .
$$

In the same way one can prove

$$
\mathbf{F}_{13}=\frac{1}{2}\left(x_{2}^{\prime} w^{\prime}-w \theta_{2}^{\prime}+\partial_{3} \boldsymbol{\kappa}_{1}\right) .
$$

To identify $\mathbf{F}_{22}$ let us observe that by the chain rule, (3.5), (3.6) and the property (d) of Theorem (3) we have

$$
\begin{aligned}
& \frac{1}{h^{3}}\left(p_{2} \partial_{2}\left(\widetilde{\boldsymbol{y}}_{2}^{h} \circ \boldsymbol{\Theta}^{h} \circ P^{h}-\boldsymbol{\Theta}_{2} \circ P^{h}\right)-p_{3} \partial_{3}\left(\widetilde{\boldsymbol{y}}_{2}^{h} \circ \boldsymbol{\Theta}^{h} \circ P^{h}-\boldsymbol{\Theta}_{2} \circ P^{h}\right)\right)= \\
& \mathbf{F}_{22}^{h}-v_{2}^{\prime} \theta_{2}^{\prime}+\mathbf{O}_{2}^{h},
\end{aligned}
$$

where $\lim _{h \rightarrow 0}\left\|\mathbf{O}_{2}^{h}\right\|_{L^{2}\left(\Omega ; \mathbb{R}^{3 \times 3}\right)}=0$. On the other hand we can conclude

$$
\begin{aligned}
& \partial_{2} \boldsymbol{\kappa}_{2}^{h}=p_{2} \partial_{2} \boldsymbol{\eta}_{2}^{h}-p_{3} \partial_{3} \boldsymbol{\eta}_{3}^{h} \\
& =\frac{1}{h^{3}}\left(p_{2} \partial_{2}\left(\widetilde{\boldsymbol{y}}_{2}^{h} \circ \boldsymbol{\Theta}^{h} \circ P^{h}-\boldsymbol{\Theta}_{2} \circ P^{h}\right)-p_{3} \partial_{3}\left(\widetilde{\boldsymbol{y}}_{2}^{h} \circ \boldsymbol{\Theta}^{h} \circ P^{h}-\boldsymbol{\Theta}_{2} \circ P^{h}\right)\right) .
\end{aligned}
$$

In the same way as before we conclude that

$$
\mathbf{F}_{22}=v_{2}^{\prime} \theta_{2}^{\prime}+\partial_{2} \boldsymbol{\kappa}_{2}
$$

Analogously we can conclude

$$
\mathbf{F}_{33}=v_{3}^{\prime} \theta_{3}^{\prime}+\partial_{3} \kappa_{3}
$$

To identify $\mathbf{F}_{23}=\mathbf{F}_{32}$ we, by using the chain rule, (3.5), (3.6) and the property (d) of Theorem (3), can conclude:

$$
\begin{aligned}
& \frac{1}{h^{3}}\left(p_{2} \partial_{2}\left(\widetilde{\boldsymbol{y}}_{3}^{h} \circ \boldsymbol{\Theta}^{h} \circ P^{h}-\boldsymbol{\Theta}_{2} \circ P^{h}\right)-p_{3} \partial_{2}\left(\widetilde{\boldsymbol{y}}_{3}^{h} \circ \boldsymbol{\Theta}^{h} \circ P^{h}-\boldsymbol{\Theta}_{2} \circ P^{h}\right)\right)= \\
& \frac{1}{h^{2}}\left(\partial_{2} \widetilde{\boldsymbol{y}}_{3}^{h}\right) \circ \boldsymbol{\Theta}^{h} \circ P^{h}-v_{2}^{\prime} \theta_{3}^{\prime}+\mathbf{O}_{3}^{h},
\end{aligned}
$$

where $\lim _{h \rightarrow 0}\left\|\mathbf{O}_{3}^{h}\right\|_{L^{2}\left(\Omega ; \mathbb{R}^{3 \times 3}\right)}=0$. In the same way we conclude

$$
\begin{aligned}
& \frac{1}{h^{3}}\left(p_{3} \partial_{2}\left(\widetilde{\boldsymbol{y}}_{2}^{h} \circ \boldsymbol{\Theta}^{h} \circ P^{h}-\boldsymbol{\Theta}_{2} \circ P^{h}\right)+p_{2} \partial_{3}\left(\widetilde{\boldsymbol{y}}_{2}^{h} \circ \boldsymbol{\Theta}^{h} \circ P^{h}-\boldsymbol{\Theta}_{2} \circ P^{h}\right)\right)= \\
& \frac{1}{h^{2}}\left(\partial_{2} \widetilde{\boldsymbol{y}}_{3}^{h}\right) \circ \boldsymbol{\Theta}^{h} \circ P^{h}-v_{3}^{\prime} \theta_{2}^{\prime}+\mathbf{O}_{4}^{h},
\end{aligned}
$$


where $\lim _{h \rightarrow 0}\left\|\mathbf{O}_{4}^{h}\right\|_{L^{2}\left(\Omega ; \mathbb{R}^{3 \times 3}\right)}=0$. It can be also concluded

$$
\begin{aligned}
p_{2} \partial_{2} \boldsymbol{\eta}_{3}^{h}-p_{3} \partial_{3} \boldsymbol{\eta}_{3}^{h}= & \frac{1}{h^{3}}\left(p_{2} \partial_{2}\left(\widetilde{\boldsymbol{y}}_{3}^{h} \circ \boldsymbol{\Theta}^{h} \circ P^{h}-\boldsymbol{\Theta}_{2} \circ P^{h}\right)\right. \\
& \left.-p_{3} \partial_{2}\left(\widetilde{\boldsymbol{y}}_{3}^{h} \circ \boldsymbol{\Theta}^{h} \circ P^{h}-\boldsymbol{\Theta}_{2} \circ P^{h}\right)\right)+\frac{1}{h^{2}} w^{h}, \\
p_{3} \partial_{2} \boldsymbol{\eta}_{2}^{h}+p_{2} \partial_{3} \boldsymbol{\eta}_{2}^{h}= & \frac{1}{h^{3}}\left(p_{3} \partial_{2}\left(\widetilde{\boldsymbol{y}}_{2}^{h} \circ \boldsymbol{\Theta}^{h} \circ P^{h}-\boldsymbol{\Theta}_{2} \circ P^{h}\right)\right. \\
& \left.+p_{2} \partial_{2}\left(\widetilde{\boldsymbol{y}}_{3}^{h} \circ \boldsymbol{\Theta}^{h} \circ P^{h}-\boldsymbol{\Theta}_{2} \circ P^{h}\right)\right)-\frac{1}{h^{2}} w^{h} .
\end{aligned}
$$

By summing the relations (4.79)-(4.82) and letting $h \rightarrow 0$ it can be concluded that

$$
2 \mathbf{F}_{23}=v_{2}^{\prime} \theta_{3}^{\prime}+v_{3}^{\prime} \theta_{2}^{\prime}+\partial_{2} \boldsymbol{\kappa}_{3}+\partial_{3} \boldsymbol{\kappa}_{2} .
$$

To prove the lower bound we can continue in the same way as in the proof of Lemma 2.3 in [25], by using the Taylor expansion, the cutting and Scorza-Dragoni theorem.

\subsection{Upper bound}

Theorem 4 (optimality of lower bound) Let $u, w \in W^{1,2}(0, L)$ and $v_{k} \in W^{2,2}(0, L)$ for $k=2,3$. Let $\boldsymbol{\gamma}$ be a function in $\mathcal{C}$ where

$$
\begin{aligned}
\mathcal{C}= & \left\{\boldsymbol{\gamma} \in L^{2}\left(\Omega ; \mathbb{R}^{3}\right): \int_{\omega} \gamma=0, \partial_{2} \gamma, \partial_{3} \gamma \in L^{2}\left(\Omega ; \mathbb{R}^{3}\right),\right. \\
& \left.\int_{\omega}\left(x_{3}^{\prime} \gamma_{2}\left(x_{1}, \cdot\right)-x_{2}^{\prime} \gamma_{3}\left(x_{1}, \cdot\right)\right) d x_{2} d x_{3}=0, \forall x_{1} \in(0, L)\right\} .
\end{aligned}
$$

Set

$$
\widetilde{\mathbf{G}}=\operatorname{sym}\left(\mathbf{J}-\frac{1}{2} \mathbf{A}^{2}+\mathbf{K}\right) .
$$

Here $\mathbf{A}, \mathbf{J}, \mathbf{K}$ are defined by the expressions (4.18), (4.59) and (4.60) and $\boldsymbol{\eta}, \boldsymbol{\kappa}$ are defined by the expressions (4.52)-(4.55).

Then there exists a sequence $\left(\hat{\boldsymbol{y}}^{h}\right) \subset W^{1,2}\left(\hat{\Omega}^{h}, \mathbb{R}^{3}\right)$ such that for $u^{h}, v_{k}^{h}, w$ defined by the expressions (4.15)-(4.17) the properties (a)-(d) of Theorem 3 are valid. Also we have that the property (f) of Theorem 3 is valid (which is equivalent that for $\boldsymbol{\eta}^{h}$ defined by the expressions (4.50)-(4.51) it is valid $\boldsymbol{\eta}^{h} \rightarrow \boldsymbol{\eta}$ weakly in $L^{2}(\Omega)$ and $\partial_{k} \boldsymbol{\eta}^{h} \rightarrow \partial_{k} \boldsymbol{\eta}$ weakly in $L^{2}(\Omega)$ ). Also the following convergence is valid

$$
\lim _{h \rightarrow 0} \frac{1}{h^{6}} \int_{\hat{\Omega}^{h}} W^{h}\left(x^{h}, \nabla \hat{\boldsymbol{y}}^{h}\right) d x^{h}=\frac{1}{2} \int_{\Omega} Q_{3}(x, \mathbf{G}(x)) d x
$$

Proof. Let us first assume that $u, w, v_{k}, \boldsymbol{\eta}$ are smooth. Then we define for $\left(x_{1}, x_{2}^{h}, x_{3}^{h}\right) \in$ $\bar{\Omega}^{h}:$

$$
\begin{aligned}
\hat{\boldsymbol{y}}^{h}\left(\boldsymbol{\Theta}^{h}\left(x_{1}, x_{2}^{h}, x_{3}^{h}\right)\right)= & \boldsymbol{\Theta}^{h}\left(x_{1}, x_{2}^{h}, x_{3}^{h}\right)+\left(\begin{array}{c}
h^{2} u\left(x_{1}\right) \\
h v_{2}\left(x_{1}\right) \\
h v_{3}\left(x_{1}\right)
\end{array}\right) \\
& +h^{2}\left(\begin{array}{c}
-x_{2}\left(v_{2}^{\prime} p_{2}+v_{3}^{\prime} p_{3}\right)\left(x_{1}\right)-x_{3}\left(v_{3}^{\prime} p_{2}-v_{2}^{\prime} p_{3}\right)\left(x_{1}\right) \\
-x_{2}\left(p_{3} w\right)\left(x_{1}\right)-x_{3}\left(p_{2} w\right)\left(x_{1}\right) \\
x_{2}\left(p_{2} w\right)\left(x_{1}\right)-x_{3}\left(p_{3} w\right)\left(x_{1}\right)
\end{array}\right) \\
& +h^{3} \boldsymbol{\eta}\left(x_{1}, \frac{x_{2}^{h}}{h}, \frac{x_{3}^{h}}{h}\right),
\end{aligned}
$$


where $\boldsymbol{\eta}: \Omega \rightarrow \mathbb{R}^{3}$ is going to be chosen later. The convergence (a)-(d) and that $\boldsymbol{\eta}^{h} \rightarrow \boldsymbol{\eta}$ weakly in $L^{2}(\Omega)$ and $\partial_{k} \boldsymbol{\eta}^{h} \rightarrow \partial_{k} \boldsymbol{\eta}$ weakly in $L^{2}(\Omega)$ can easily seen to be valid for this sequence. We also have

$$
\begin{aligned}
\nabla \hat{\boldsymbol{y}}^{h} \nabla \boldsymbol{\Theta}^{h}= & \nabla \boldsymbol{\Theta}^{h}+\left(\begin{array}{cc|c}
h^{2} u^{\prime} & -h\left(v_{2}^{\prime} p_{2}+v_{3}^{\prime} p_{3}\right) & -h\left(v_{3}^{\prime} p_{2}-v_{2}^{\prime} p_{3}\right) \\
h v_{2}^{\prime} & -h p_{3} w & -h p_{2} w \\
h v_{3}^{\prime} & h p_{2} w & -h p_{3} w
\end{array}\right) \\
& +h^{2}\left(\begin{array}{c}
-x_{2}\left(v_{2}^{\prime} p_{2}+v_{3}^{\prime} p_{3}\right)^{\prime}+x_{3}\left(v_{2}^{\prime} p_{3}-v_{3}^{\prime} p_{2}\right)^{\prime} \\
-x_{2}\left(p_{3} w\right)^{\prime}-x_{3}\left(p_{2} w\right)^{\prime} \\
x_{2}\left(p_{2} w\right)^{\prime}-x_{3}\left(p_{3} w\right)^{\prime}
\end{array}\left|\partial_{2} \boldsymbol{\eta}\right| \partial_{3} \boldsymbol{\eta}\right) \\
& +O\left(h^{3}\right) .
\end{aligned}
$$

From (4.88), by using (3.8), we conclude

$$
\begin{aligned}
\nabla \hat{\boldsymbol{y}}^{h}= & \mathbf{I}+h\left(\begin{array}{ccc}
0 & -v_{2}^{\prime} & -v_{3}^{\prime} \\
v_{2}^{\prime} & 0 & -w \\
v_{3}^{\prime} & w & 0
\end{array}\right) \\
& +h^{2}\left(\begin{array}{ccc}
u^{\prime}+v_{2}^{\prime} \theta_{2}^{\prime}+v_{3}^{\prime} \theta_{3}^{\prime} & 0 & 0 \\
w \theta_{3}^{\prime} & v_{2}^{\prime} \theta_{2}^{\prime} & v_{2}^{\prime} \theta_{3}^{\prime} \\
-w \theta_{2}^{\prime} & v_{3}^{\prime} \theta_{2}^{\prime} & v_{3}^{\prime} \theta_{3}^{\prime}
\end{array}\right) \\
& +h^{2}\left(\begin{array}{c}
-x_{2}\left(v_{2}^{\prime \prime} p_{2}+v_{3}^{\prime \prime} p_{3}\right)+x_{3}\left(v_{2}^{\prime \prime} p_{3}-v_{3}^{\prime \prime} p_{2}\right) \\
-x_{2}\left(p_{3} w^{\prime}\right)-x_{3}\left(p_{2} w^{\prime}\right) \\
x_{2}\left(p_{2} w^{\prime}\right)-x_{3}\left(p_{3} w^{\prime}\right)
\end{array}\left|\partial_{2} \kappa\right| \partial_{3} \kappa\right) \\
& +O\left(h^{3}\right) .
\end{aligned}
$$

Using the identity $(\mathbf{I}+\mathbf{M})^{T}(\mathbf{I}+\mathbf{M})=\mathbf{I}+2 \operatorname{sym} \mathbf{M}+\mathbf{M}^{T} \mathbf{M}$ we obtain

$$
\left(\nabla \hat{\boldsymbol{y}}^{h}\right)^{T}\left(\nabla \hat{\boldsymbol{y}}^{h}\right)=\mathbf{I}+2 h^{2} \operatorname{sym} \mathbf{J}+2 h^{2} \operatorname{sym} \mathbf{K}+h^{2} \mathbf{A}^{T} \mathbf{A}+O\left(h^{3}\right),
$$

where $\left\|O\left(h^{3}\right)\right\|_{L^{\infty}\left(\Omega ; \mathbb{R}^{3 \times 3}\right)} \leq C h^{3}$, for some $C>0$.

Taking the square root we obtain

$$
\left[\left(\nabla \hat{\boldsymbol{y}}^{h}\right)^{T}\left(\nabla \hat{\boldsymbol{y}}^{h}\right)\right]^{1 / 2}=\mathbf{I}+h^{2} \widetilde{\mathbf{G}}+O\left(h^{3}\right) .
$$

We have $\operatorname{det}\left(\nabla \hat{\boldsymbol{y}}^{h}\right)>0$ for sufficiently small $h$. Hence by frame-indifference $\left.W\left(x,\left(\nabla \hat{\boldsymbol{y}}^{h}\right) \circ \boldsymbol{\Theta}^{h} \circ P^{h}\right)=W\left(x,\left[\nabla \hat{\boldsymbol{y}}^{h}\right)^{T}\left(\nabla \hat{\boldsymbol{y}}^{h}\right)\right]^{1 / 2} \circ \boldsymbol{\Theta}^{h} \circ P^{h}\right)$; thus by (4.90) and Taylor expansion we obtain:

$$
\frac{1}{h^{4}} W\left(x,\left(\nabla \hat{\boldsymbol{y}}^{h}\right) \circ \boldsymbol{\Theta}^{h} \circ P^{h}\right) \rightarrow \frac{1}{2} Q_{3}(x, \widetilde{\mathbf{G}}(x)) \text { a.e }
$$

and by the property ii) of $W$ for $h$ small enough

$$
\frac{1}{h^{4}} W\left(x,\left(\nabla \hat{\boldsymbol{y}}^{h}\right) \circ \boldsymbol{\Theta}^{h} \circ P^{h}\right) \leq \frac{1}{2} C\left(\|\mathbf{J}\|^{2}+\|\mathbf{K}\|^{2}+\|\mathbf{A}\|^{4}\right)+C h .
$$

The equality (4.86) follows by the dominated convergence theorem. Namely, we have

$$
\begin{aligned}
\frac{1}{h^{6}} \int_{\hat{\Omega}^{h}} W^{h}\left(x, \nabla \hat{\boldsymbol{y}}^{h}\right) d x= & \frac{1}{h^{4}} \int_{\Omega} W\left(x,\left(\nabla \hat{\boldsymbol{y}}^{h}\right) \circ \boldsymbol{\Theta}^{h} \circ P^{h}\right) d x \\
& \rightarrow \frac{1}{2} \int_{\Omega} Q_{3}(x, \widetilde{\mathbf{G}}) d x .
\end{aligned}
$$


In the general case, it is enough to smoothly approximate $u, w$ in the strong topology of $W^{1,2}, v_{k}$ in the strong topology of $W^{2,2}$, and $\boldsymbol{\eta}, \partial_{k} \boldsymbol{\eta}$ in the strong topology of $L^{2}$ and to use the continuity of the right hand side of (4.86) with respect to these convergences.

Remark 6 Notice that

$$
\mathbf{K}=\left(\mathbf{A}^{\prime}\left(\begin{array}{c}
0 \\
x_{2}^{\prime} \\
x_{3}^{\prime}
\end{array}\right)\left|\partial_{2} \boldsymbol{\kappa}\right| \partial_{3} \boldsymbol{\kappa}\right)=\mathbf{L}+\left(\mathbf{A}^{\prime}\left(\begin{array}{c}
0 \\
x_{2}^{\prime} \\
x_{3}^{\prime}
\end{array}\right)\left|\partial_{2} \boldsymbol{\beta}\right| \partial_{3} \boldsymbol{\beta}\right)
$$

Here $\boldsymbol{\beta}=\boldsymbol{\gamma} \circ\left(\boldsymbol{x}^{\prime}\right)^{-1}$ and

$$
\mathbf{L}=\left(\begin{array}{ccc}
0 & 0 & 0 \\
0 & g_{2}^{2} & g_{2}^{3} \\
0 & g_{3}^{2} & g_{3}^{3}
\end{array}\right)
$$

From the fact that $\boldsymbol{\gamma} \in \mathcal{C}$ we can conclude $\boldsymbol{\beta} \in \mathcal{B}$, where

$$
\begin{aligned}
& \mathcal{B}=\left\{\boldsymbol{\beta} \in L^{2}\left(\Omega^{\prime} ; \mathbb{R}^{3}\right): \int_{\omega} \boldsymbol{\beta}=0, \partial_{2} \boldsymbol{\beta}, \partial_{3} \boldsymbol{\beta} \in L^{2}\left(\Omega^{\prime} ; \mathbb{R}^{3}\right),\right. \\
& \int_{\omega^{\prime}\left(x_{1}\right)}\left(x_{3}^{\prime} \boldsymbol{\beta}_{2}\left(x_{1}, \cdot\right)-x_{2}^{\prime} \boldsymbol{\beta}_{3}\left(x_{1}, \cdot\right) d x_{2}^{\prime} d x_{3}^{\prime}=0, \text { for a.e. } x_{1} \in(0, L)\right\} .
\end{aligned}
$$

4.3 Identification of the $\Gamma$-limit

Let $Q:(0, L) \times \mathbb{R} \times \operatorname{so}(3) \rightarrow[0,+\infty)$ be defined as

$$
\begin{aligned}
& Q\left(x_{1}, t, \mathbf{F}\right)= \\
& \min _{\boldsymbol{\alpha} \in W^{1,2}\left(\omega^{\prime}\left(x_{1}\right) ; \mathbb{R}^{3}\right)} \int_{\omega^{\prime}\left(x_{1}\right)} Q_{3}\left(x,\left(\mathbf{F}\left(\begin{array}{c}
0 \\
x_{2}^{\prime} \\
x_{3}^{\prime}
\end{array}\right)+t \boldsymbol{e}_{1}\left|\partial_{2} \boldsymbol{\alpha}\right| \partial_{3} \boldsymbol{\alpha}\right)\right) d x_{2}^{\prime} d x_{3}^{\prime},
\end{aligned}
$$

where $Q_{3}$ is the quadratic form defined in (4.61). For $u, w \in W^{1,2}(0, L)$ and $v_{2}, v_{3} \in$ $W^{2,2}(0, L)$ we introduce the functional

$$
I^{0}\left(u, v_{2}, v_{3}, w\right):=\frac{1}{2} \int_{0}^{L} Q\left(x_{1}, u^{\prime}+v_{2}^{\prime} \theta_{2}^{\prime}+v_{3}^{\prime} \theta_{3}^{\prime}+\frac{1}{2}\left(\left(v_{2}^{\prime}\right)^{2}+\left(v_{3}^{\prime}\right)^{2}\right), \partial_{1} \mathbf{A}\right) d x_{1},
$$

where $\mathbf{A} \in W^{1,2}((0, L)$; so(3)) is defined by (4.18). We shall state the result of $\Gamma$ convergence of the functionals $\frac{1}{h^{4}} I^{h}$ to $I^{0}$. Before stating the theorem we analyze some properties of the limit density $Q$.

Remark 7 By using the remarks in the beginning of chapter 4 in [25] the following facts can be concluded:

a) The functional $Q_{3}(x, \mathbf{G})$ is coercive on symmetric matrices i.e. there exists a constant $C>0$, independent of $x$, such that $Q_{3}(x, \mathbf{G}) \geq C\|\operatorname{sym} \mathbf{G}\|^{2}$, for every $\mathbf{G}$ (this is the direct consequence of the assumption iv) on $W$ ). The minimum in (4.93) is attained. Since the functional $Q_{3}(x, \mathbf{G})$ depends only on the symmetric 
part of $\mathbf{G}$, it is invariant under transformation $\boldsymbol{\alpha} \mapsto \boldsymbol{\alpha}+c_{1}+c_{2}\left(x^{\prime}\right)^{\perp}$ and hence the minimum can be computed on the subspace

$$
\begin{aligned}
& V_{x_{1}}:=\left\{\boldsymbol{\alpha} \in W^{1,2}\left(\omega^{\prime}\left(x_{1}\right), \mathbb{R}^{3}\right):\right. \int_{\omega^{\prime}\left(x_{1}\right)} \boldsymbol{\alpha}=0, \\
&\left.\int_{\omega^{\prime}\left(x_{1}\right)}\left(x_{3}^{\prime} \boldsymbol{\alpha}_{2}-x_{2}^{\prime} \boldsymbol{\alpha}_{3}\right) d x_{2}^{\prime} d x_{3}^{\prime}=0\right\} .
\end{aligned}
$$

Strict convexity of $Q_{3}(x, \cdot)$ on symmetric matrices ensures that the minimizer is unique in $V_{x_{1}}$.

b) Fix $x_{1} \in(0, L), t \in \mathbb{R}$ and $\mathbf{F} \in \operatorname{so}(3)$. Let $\boldsymbol{\alpha}^{\text {min }} \in V$ be the unique minimizer of the problem (4.93). We set

$$
g\left(x_{2}^{\prime}, x_{3}^{\prime}\right)=\mathbf{F}\left(\begin{array}{c}
0 \\
x_{2}^{\prime} \\
x_{3}^{\prime}
\end{array}\right)+t \boldsymbol{e}_{1}, \quad b_{i j}^{h k}=\frac{\partial^{2} W}{\partial \mathbf{F}_{i h} \partial \mathbf{F}_{j k}}(x, \mathbf{I}),
$$

and we call $B^{h k}$ the matrix in $\mathbb{R}^{3 \times 3}$ whose elements are given by $\left(B^{h k}\right)_{i j}=b_{i j}^{h k}$. Then $\boldsymbol{\alpha}^{\text {min }}$ satisfies the following Euler-Lagrange equation:

$$
\int_{\omega^{\prime}\left(x_{1}\right)} \sum_{h, k=2,3}\left(B^{h k} \partial_{k} \boldsymbol{\alpha}^{m i n}, \partial_{h} \varphi\right) d x_{2}^{\prime} d x_{3}^{\prime}=-\int_{\omega^{\prime}\left(x_{1}\right)} \sum_{h=2,3}\left(B^{h 1} g, \partial_{h} \varphi\right) d x_{2}^{\prime} d x_{3}^{\prime},
$$

for every $\varphi \in W^{1,2}\left(\omega^{\prime}\left(x_{1}\right) ; \mathbb{R}^{3 \times 3}\right)$. From this equation it is clear that $\boldsymbol{\alpha}^{\text {min }}$ depends linearly on $(t, \mathbf{F})$. Moreover $Q$ is uniformly positive definite, i.e.

$$
Q\left(x_{1}, t, \mathbf{F}\right) \geq C\left(t^{2}+\|\mathbf{F}\|^{2}\right), \quad \forall t \in \mathbb{R}, \forall \mathbf{F} \in \operatorname{so}(3),
$$

and the constant $C$ does not depend on $x_{1}$.

c) By mimicking the proof of Remark 4.3 in [25] it can be seen that there exists a constant $C^{\prime}$ (independent of $x_{1}, t$ and $\mathbf{F}$ ) such that

$$
\left\|\partial_{2} \boldsymbol{\alpha}^{\min }\right\|_{L^{2}\left(\omega^{\prime}\left(x_{1}\right) ; \mathbb{R}^{3 \times 3}\right)}+\left\|\partial_{3} \boldsymbol{\alpha}^{\min }\right\|_{L^{2}\left(\omega^{\prime}\left(x_{1}\right) ; \mathbb{R}^{3 \times 3}\right)} \leq C^{\prime}\|g\|_{L^{2}\left(\omega^{\prime}\left(x_{1}\right) ; \mathbb{R}^{3 \times 3}\right)}^{2},
$$

for a.e. $x_{1} \in(0, L)$. To adapt the proof we only need to have that the constant in the Korn's inequality

$$
\int_{\omega^{\prime}\left(x_{1}\right)} \sum_{j, k=2,3}\left|\partial_{k} \boldsymbol{\alpha}_{j}^{m i n}\right|^{2} d x_{2}^{\prime} d x_{3}^{\prime} \leq C_{1} \int_{\omega^{\prime}\left(x_{1}\right)} \sum_{j, k=2,3}\left|e_{j k}\left(\boldsymbol{\alpha}^{m i n}\right)\right|^{2} d x_{2}^{\prime} d x_{3}^{\prime}
$$

can be chosen independently of $x_{1}$. This is proved in Lemma 1 .

d) When $Q_{3}$ does not depend on $x_{2}, x_{3}$ we can find a more explicit representation for $Q$. More precisely $Q$ can be decomposed into the sum of two quadratic forms

$$
Q\left(x_{1}, t, \mathbf{F}\right)=Q_{1}\left(x_{1}, t\right)+Q_{2}\left(x_{1}, \mathbf{F}\right),
$$

where

$$
\begin{aligned}
Q_{1}\left(x_{1}, t\right) & :=\min _{\boldsymbol{a}, \boldsymbol{b} \in \mathbb{R}^{3}} Q_{3}\left(x_{1},\left(t \boldsymbol{e}_{1}|\boldsymbol{a}| \boldsymbol{b}\right)\right), \\
Q_{2}\left(x_{1}, 0, \mathbf{F}\right) & :=Q\left(x_{1}, 0, \mathbf{F}\right) .
\end{aligned}
$$


The relations (4.8) are only needed for this. If we assume the isotropic and homogenous case i.e.

$$
Q_{3}(\mathbf{F})=2 \mu\left|\frac{\mathbf{F}+\mathbf{F}^{T}}{2}\right|^{2}+\lambda(\operatorname{trace} \mathbf{F})^{2}
$$

then after some calculation (see Remark 3.5 in [24]) it can be shown that

$$
\begin{aligned}
Q_{1}(t)= & \frac{\mu(3 \lambda+2 \mu)}{\lambda+\mu} t^{2} \\
Q_{2}\left(x_{1}, \mathbf{F}\right)= & \frac{\mu(3 \lambda+2 \mu)}{\lambda+\mu}\left(\mathbf{F}_{12} \int_{\omega^{\prime}\left(x_{1}\right)}\left(x_{2}^{\prime}\right)^{2} d x_{2}^{\prime} d x_{3}^{\prime}\right. \\
& \left.+2 \mathbf{F}_{12} \mathbf{F}_{13} \int_{\omega^{\prime}\left(x_{1}\right)} x_{2}^{\prime} x_{3}^{\prime} d x_{2}^{\prime} d x_{3}^{\prime}+\mathbf{F}_{13} \int_{\omega^{\prime}\left(x_{1}\right)}\left(x_{3}^{\prime}\right)^{2} d x_{2}^{\prime} d x_{3}^{\prime}\right) \\
& +\mu \tau \mathbf{F}_{23},
\end{aligned}
$$

where the constant $\tau$ is so-called torsional rigidity, defined as

$$
\tau\left(\omega^{\prime}\left(x_{1}\right)\right)=\tau(\omega)=\int_{\omega}\left(x_{2}^{2}+x_{3}^{2}-x_{2} \partial_{3} \varphi+x_{3} \partial_{2} \varphi\right) d x_{2} d x_{3},
$$

and $\varphi$ is the torsion function i.e. the solution of the Neumann problem

$$
\begin{cases}\triangle \varphi=0 & \text { in } \omega \\ \partial_{\nu} \varphi=-\left(x_{3},-x_{2}\right) \cdot \nu & \text { on } \partial \omega\end{cases}
$$

The following theorem can be proved in the same way as Theorem 4.5 in [23] (we need Theorem 3, Lemma 2, Theorem 4, Remark 6 and Remark 7).

Theorem 5 As $h \rightarrow 0$, the functionals $\frac{1}{h^{4}} I^{h}$ are $\Gamma$-convergent to the functional $I^{0}$ given in (4.94), in the following sense:

i) (compactness and liminf inequality) if $\limsup _{h \rightarrow 0} h^{-4} I^{h}<+\infty$ then there exists constants $\overline{\mathbf{R}}^{h} \in \mathrm{SO}(3), c^{h} \in \mathbb{R}^{3}$ such that (up to subsequences) $\overline{\mathbf{R}}^{h} \rightarrow \overline{\mathbf{R}}$ and the functions defined by

$$
\begin{aligned}
\widetilde{\boldsymbol{y}}^{h} & :=\left(\overline{\mathbf{R}}^{h}\right)^{T} \boldsymbol{y}^{h}-c^{h}, \quad u^{h}=\frac{1}{A} \int_{\omega} \frac{\widetilde{\boldsymbol{y}}_{1}^{h} \circ \boldsymbol{\Theta}^{h} \circ P^{h}-x_{1}}{h^{2}} d x_{2} d x_{3} \\
v_{k}^{h} & =\frac{1}{A} \int_{\omega} \frac{\widetilde{\boldsymbol{y}}_{k}^{h} \circ \boldsymbol{\Theta}^{h} \circ P^{h}-h \theta_{k}}{h} d x_{2} d x_{3} \\
w^{h} & =\frac{1}{A \mu(\omega)} \int_{\omega} \frac{x_{2}^{\prime}\left(\widetilde{\boldsymbol{y}}_{3} \circ \boldsymbol{\Theta}^{h} \circ P^{h}\right)-x_{3}^{\prime}\left(\widetilde{\boldsymbol{y}}_{2} \circ \boldsymbol{\Theta}^{h} \circ P^{h}\right)}{h^{2}} d x_{2} d x_{3}
\end{aligned}
$$

satisfy

(a) $\left(\nabla \widetilde{\boldsymbol{y}}^{h}\right) \circ \boldsymbol{\Theta}^{h} \circ P^{h} \rightarrow \mathbf{I}$ in $L^{2}(\Omega)$.

(b) there exist $u, w \in W^{1,2}(0, L)$ such that $u^{h} \rightarrow u$ and $w^{h} \rightarrow w$ weakly in $W^{1,2}(0, L)$.

(c) there exists $v_{k} \in W^{2,2}(0, L)$ such that $v_{k}^{h} \rightarrow v_{k}$ strongly in $W^{1,2}(0, L)$ for $k=2,3$.

Moreover we have

$$
\liminf _{h \rightarrow 0} \frac{1}{h^{4}} I^{h}\left(\boldsymbol{y}^{h}\right) \geq I^{0}\left(u, v_{2}, v_{3}, w\right) .
$$


ii) (limsup inequality) for every $v, w \in W^{1,2}(0, L), v_{2}, v_{3} \in W^{2,2}(0, L)$ there exists $\left(\hat{\boldsymbol{y}}^{h}\right)$ such that (a)-(c) hold (with $\widetilde{\boldsymbol{y}}^{h}$ replaced by $\hat{\boldsymbol{y}}^{h}$ ) and

$$
\lim _{h \rightarrow 0} \frac{1}{h^{4}} I^{h}\left(\hat{\boldsymbol{y}}^{h}\right)=I^{0}\left(u, v_{2}, v_{3}, w\right)
$$

Remark 8 Let $f_{2}, f_{3} \in L^{2}(0, L)$. We introduce the functional

$$
J^{0}=I^{0}\left(u, v_{2}, v_{3}, w\right)-\int_{0}^{L} \sum_{k=2,3} f_{k} v_{k}
$$

for every $u \in W^{1,2}(0, L), v_{2}, v_{3} \in W^{2,2}(0, L)$, and $w \in W^{1,2}(0, L)$. The functional $J^{0}$ can be obtained as $\Gamma$-limit of the energies $\frac{1}{h^{4}} I^{h}$ by adding a term describing transversal body forces of order $h^{3}$ (see [13], see also [32]). For longitudinal body forces see [18]. The problem for longitudinal body forces arises because the longitudinal forces should be of order $h^{2}$, the same order as for the model in [24]. One needs to impose certain stability condition to see which model describes the behavior of the body for the longitudinal forces of order $h^{2}$.

Remark 9 The term $u^{\prime}+v_{2}^{\prime} \theta_{2}^{\prime}+v_{3}^{\prime} \theta_{3}^{\prime}+\frac{1}{2}\left(\left(v_{2}^{\prime}\right)^{2}+\left(v_{3}^{\prime}\right)^{2}\right)$ in the strain measures the extension of the central line (which is of the second order). Namely, if we approximate the deformation of the weakly curved rod by:

$$
\begin{aligned}
& \varphi_{1}\left(x_{1}, x_{2}, x_{3}\right)=x_{1}+h^{2} u+h^{2} x_{2}^{\prime}\left(v_{2}^{\prime}+\theta_{2}^{\prime}\right)+h^{2} x_{3}^{\prime}\left(v_{3}^{\prime}+\theta_{3}^{\prime}\right) \\
& \varphi_{k}\left(x_{1}, x_{2}, x_{3}\right)=h \theta_{k}+h x_{k}^{\prime}+h v_{k}+h^{2}\left(x_{k}^{\prime}\right)^{\perp} w, \text { for } k=2,3,
\end{aligned}
$$

we see, that it is valid

$$
\begin{aligned}
\left\|\partial_{1} \varphi\left(x_{1}, 0,0\right)\right\|^{2}-\left\|\partial_{1} \Theta^{h}\left(x_{1}, 0,0\right)\right\|^{2}= & h^{2}\left(2 u^{\prime}+2 v_{2}^{\prime} \theta_{2}^{\prime}+2 v_{3}^{\prime} \theta_{3}^{\prime}\right. \\
& \left.+\left(v_{2}^{\prime}\right)^{2}+\left(v_{3}^{\prime}\right)^{2}\right) .
\end{aligned}
$$

Remark 10 The existence of the solution for the functional $J^{0}$ under the Dirichlet boundary condition for $v_{k}$ at both ends of the rod can be proved directly. It is also enough that we impose $v_{2}, v_{2}^{\prime}, v_{3}, v_{3}^{\prime}$ at the one end. The existence can also be proved for the free boundary condition under the hypothesis that $\int_{0}^{L} f_{k} d x_{1}=0$, $\int_{0}^{L} x_{1} \boldsymbol{f}_{k} d x_{1}=0$ for $k=2,3$. It can be done in the same way as the proof of Lemma 5 in [32].

\section{References}

1. Acerbi, E., Butazzo, G., Percivale, D.: A variational definition of the strain energy for an elastic string, Journal of Elasticity, 25, 137-148 (1991)

2. Adams, R.A.: Sobolev spaces, Academic press, New York 1975.

3. Alvarez-Dios, J.A. , Viano, J.M.: A bending and stretching asymptotic theory for general elastic shallow arches, ESAIM: Proc., Vol. 2, 145-152 (1997)

4. Alvarez-Dios, J.A. , Viano, J.M.: Mathematical Justication of a One-dimensional Model for General Elastic Shallow Arches, Mathematical Methods in the Applied Sciences, Volume 21, Issue 4, 281-325 (1998)

5. S.S. Antman, Nonlinear problems of elasticity. Second edition, Applied Mathematical Sciences, 107, Springer, New York, 2005. 
6. A. Braides: $\Gamma$-convergence for Beginners, Oxford University Press, Oxford, 2002.

7. Ciarlet, P.G.: Mathematical elasticity. Vol. I, Three-dimensional elasticity, North-Holland Publishing Co., Amsterdam, 1988.

8. Ciarlet, P.G.: Mathematical elasticity. Vol. II. Theory of plates. Studies in Mathematics and its Applications, 27. North-Holland Publishing Co., Amsterdam (1997).

9. Ciarlet, P.G.: Mathematical elasticity. Vol. III. Theory of shells. Studies in Mathematics and its Applications, 29. North-Holland Publishing Co., Amsterdam (2000).

10. Dal Maso, G.: An introduction to $\Gamma$-convergence, Progress in Nonlinear Differential Equations and Their Applications, Birkäuser, Basel (1993).

11. Fox, D.D., Raoult A., Simo, J.C.: A justification of nonlinear properly invariant plate theories, Arch. Rational Mech. Anal., 124, p. 157199 (1993).

12. Friesecke, G., James R.D., Müler, S.: A theorem on geometric rigidity and the derivation of nonlinear plate theory from three-dimensional elasticity, Comm. Pure Appl. Math. 55, 1461-1506 (2002)

13. Friesecke, G., James R.D., Müler, S.: A Hierarchy of Plate Models Derived from Nonlinear Elasticity by $\Gamma$-Convergence, Archive for Rational Mechanics and Analysis 180, no.2, $183-236(2006)$.

14. Friesecke, G., James R.D., Müler, S.: The Föppl-von Kármán plate theory as a low energy $\Gamma$-limit of nonlinear elasticity, Comptes Rendus Mathematique 335, no. 2, 201-206 (2002).

15. Friesecke, G., James R., Mora, M.G., Müller, S.: Derivation of nonlinear bending theory for shells from three-dimensional nonlinear elasticity by $\Gamma$-convergence, C. R. Math. Acad. Sci. Paris, 336, no. 8, 697-702 (2003).

16. Le Dret, H., Raoult, A.: The nonlinear membrane model as a variational limit of nonlinear three-dimensional elasticity, Journal de Mathématiques Pures et Appliquées 74, 549-578 (1995).

17. Le Dret, H., Raoult, A.: The membrane shell model in nonlinear elasticity: A variational asymptotic derivation, Journal of Nonlinear Science 6, Number 1, 59-84 (1996).

18. Lecumberry, M., Müller, S.: Stability of slender bodies under compression and validity of the von Kármán theory, Archive for Rational Mechanics and Analysis, Volume 193, Number 2, 255-310 (2009).

19. Lewicka, M., Mora, M.G., Pakzad, M.: Shell theories arising as low energy $\Gamma$-limit of $3 \mathrm{~d}$ nonlinear elasticity, Ann. Scuola Norm. Sup. Pisa Cl. Sci. 5, Vol. IX, 1-43 (2010).

20. Lewicka, M., Mora, M.G., Pakzad, M.: The matching property of infinitesimal isometries on elliptic surfaces and elasticity of thin shells, accepted in Arch. Rational Mech. Anal.

21. Lewicka, M., Pakzad, M.: The infinite hierarchy of elastic shell models: some recent results and a conjecture, accepted in Fields Institute Communications (2010).

22. Marigo, J.J., Meunier, N.: Hierarchy of One-Dimensional Models in Nonlinear Elasticity, Journal of Elasticity, 83, 1-28 (2006)

23. Mora, M.G., Scardia, L.: Convergence of equilibria of thin elastic plates under physical growth conditions for the energy density, submitted paper.

24. Mora, M.G., Müller, S.: Derivation of the nonlinear bending-torsion theory for inextensible rods by Gamma-convergence, Calc. Var., 18, 287-305 (2003)

25. Mora, M.G., Müller, S.: A nonlinear model for inextensible rods as a low energy Gammalimit of three-dimensional nonlinear elasticity, Ann. Inst. H. Poincar Anal. Nonlin., 21, 271-293 (2004)

26. Mora, M.G., Müller, S., Schultz, M.G.: Convergence of equilibria of planar thin elastic beams, Indiana Univ. Math. J., 56, 2413-2438 (2007)

27. Mora, M.G., Müller, S.: Convergence of equilibria of three-dimensional thin elastic beams, Proc. Roy. Soc. Edinburgh Sect. A 138, 873-896 (2008)

28. Müller, S., Packzad, M.R.: Convergence of equilibria of thin elastic plates : the von Kármán case, Communications in Partial Differential Equations 33, Number 6, 1018 1032 (2008)

29. Oleinik, O.A., Shamaev, A.S., Yosifian, G.A.: Mathematical problems in elasticity and homogenization, North-Holland, 1992.

30. Scardia, L.: The nonlinear bending-torsion theory for curved rods as Gamma-limit of three-dimensional elasticity, Asymptot. Anal., 47, 317-343 (2006)

31. Scardia, L.: Asymptotic models for curved rods derived from nonlinear elasticity by $\Gamma$ convergence, submitted paper.

32. Velcic, I.: Shallow shell models by $\Gamma$-convergence, submitted paper. Preprint http://web.math.hr/ ivelcic

33. Ziemer, W.: Weakly Differentiable Functions, Springer-Verlag: New York (1989). 